\title{
Line structure representation for road network analysis
}

\author{
Stephen Marshall \\ University College London \\ s.marshall@ucl.ac.uk
}

\begin{abstract}
Road hierarchy and network structure are intimately linked; however, there is not a consistent basis for representing and analyzing the particular hierarchical nature of road network structure. This paper introduces the line structureidentified mathematically as a kind of linearly ordered incidence structure-as a means of representing road network structure and demonstrates its relation to existing representations of road networks: the "primal" graph, the "dual" graph, and the route structure. In doing so, the paper shows how properties of continuity, junction type, and hierarchy relating to differential continuity and termination are necessarily absent from primal and dual graph representations but intrinsically present in line structure representations. A new property indicative of hierarchical status"cardinality"-is introduced and illustrated with application to example networks. The paper concludes by highlighting newly explicit relationships between different kinds of road network structure representation.
\end{abstract}

Keywords: network science, road hierarchy, route structure, graph theory, line structure, cardinality

\section{Article history:}

Received: March 5, 2014

Accepted: October 13, 2014

Available online: July 1,

2015

Introduction

Road networks are routinely interpreted in terms of configurational structures such as "trees" or "grids," but network structure can also be interpreted in terms of hierarchical relations: between main and subsidiary, strategic and local, or through and side roads. In fact, these two different kinds of structure-relating to "configuration" and "constitution" (Marshall 2005) —are in some ways related. Yet despite the proliferation of studies of road networks, there is not a consistent basis for representing and analyzing this dual nature of network structure, either within traditions of network science or network design and management.

On the one hand, broadly speaking, there are "network science" approaches concerned with understanding the structure of networks, whether through empirical studies (e.g., Masucci et al. 2009; Strano et al. 2012) or modeling (e.g., Yerra and Levinson 2005; Barthélemy and Flammini 2008). These capture network properties such as average degree, clustering coefficient, average shortest path, meshedness, betweenness centrality, webness or treeness (e.g., Claramunt and Winter 2007; Barthélemy 2011; Xie and Levinson 2011), or general graph theoretic measures such as alpha index, beta index, and so on (e.g., Kansky 1963; Haggett and Chorley 1969; Xie and Levinson 2007). However, in general, these approaches tend to be "node-centric" rather than "link-centric" (Erat, Löchl, and Axhausen 2008; Xie and Levinson 2011), and less attention is given to capturing measures of hierarchy arising from the differential continuity and termination of roads through junctions. This concerns the difference in status between a main road and a side road, whereby the "main-ness" of the former is due to its prevailing through its junction with the latter, while the "side-ness" of the latter is due

Copyright 2015 Stephen Marshall

http://dx.doi.org/10.5198/jtlu.2015.744

ISSN: 1938-7849 | Licensed under the Creative Commons Attribution - Noncommercial License 3.0

The Journal of Transport and Land Use is the official journal of the World Society for Transport and Land Use (WSTLUR) and is published and sponsored by the University of Minnesota Center for Transportation Studies. This paper is also published with sponsorship from WSTLUR and the Institutes of Transportation Studies at University of California, Davis and University of California, Berkeley. 
to its yielding to the former. Barthélemy's recent extensive review of network analysis (2011) does not address hierarchical properties or relations of this kind-that paper's focus lies elsewhere-but hierarchy of this kind is central to this paper.

On the other hand, road hierarchy is of definite concern to road network design and management (Jones 1986; Brindle 1996; Jones, Boujenko, and Marshall 2007). However, conventional road management approaches have given less explicit attention to capturing hierarchical structure quantitatively. While tiers in the hierarchy may be labeled numerically (e.g., I, II, III), the articulation of structure itself has tended to rely on graphic depiction (for example, MoT 1963; AASHTO 2001; Essex County Council 1973; DfT and DCLG 2007; Jones, Boujenko, and Marshall 2007) or use of loose descriptive terms (e.g., "hierarchical" versus "non hierarchical," Marshall 2005).

Commonly agreed indicators pinpointing different kinds of hierarchical structure are lacking. While hierarchical structure can already be inferred in "route structure" analysis, or explicitly categorized in terms of "constitutional structure" (Marshall 2005), the link between these two has not been fully explored.

This leaves a number of outstanding issues for resolution. It is not completely clear how different ways of representing networks (such as via graphs or route structures) relate to each other, or how these relate to different kinds of structure (e.g., "configurational" versus "constitutional" structure) or to properties that could distinguish "more hierarchical" versus "less hierarchical" structures. Accordingly, this paper sets out to clarify, deepen, and integrate understanding of the different kind of network representations and their hierarchical properties and to provide a more fully developed way of capturing road network structure.

This paper first sets out key issues for the representation of networks (Section 2). In Section 3, the line structure as a means of network representation is introduced, and then related to graphs and other representations in Section 4. Section 5 discusses properties captured by the different representations and the information (matrix size) required to specify them, and Section 6 demonstrates application to small example road networks. Section 7 draws conclusions on the significance of these findings and suggests future research and application.

\section{Representations of road network structure}

This section sets out the scope of terms used (2.1), then addresses what aspects of the road system are to be represented (2.2) and how those aspects are to be represented (2.3).

\subsection{Use of terms}

In this paper, the terms road and road network will be used throughout, for the sake of consistency, though, in many applications these roads will be streets, or they could be footpaths or other kinds of linear elements where continuity through intersection of elements may be significant. The term road system is generally used to refer to roads in the most general sense (including their physical fabric); road layout refers to the twodimensional aspect (including distances, widths, curvature, etc.), and network to its one-dimensional aspect (links and nodes, etc.). The term junction is used in the road network context to refer to any connection point between different roads, while intersection is generally used in the mathematical context to refer to any connections between lines, sets, or other mathematical elements.

The term line generally refers to a linear graphical element (whether curved or straight) used to represent a road or other linear real-world feature, which could in practice be a road center-line, or even a bus "line" (service). The term route refers to the kind of linear element representing a road in route structure analysis (Section 2.3.5). Any graph can be described in terms of vertices and edges; these may also be referred to as nodes 
and links in the so-called "primal" form of representation in the context of road network diagrams. The "primal" and "dual" graph representations are discussed in more detail in section 2.3.1. ${ }^{1}$

\subsection{Elements for representation}

When considering aspects of a road system to be represented, there will always be some degree of interpretation, and selective consideration of "what is being mapped" in the context of some social or functional purpose (Godehardt 1990; Peponis et al. 1998; Buckwalter 2001; Hillier and Penn 2004; Turner, Penn, and Hillier 2005; Marshall 2005; Turner 2007; Batty 2008; Batty 2010). Normally road network analysis abstracts from a two-dimensional planar representation of the road layout—a map-or a linear component thereof (Courtat, Gloaguen, and Douady 2011). The question becomes how to get from two- or onedimensional geometry to a topological representation suitable for structural analysis.

A road layout representation may be based on road center-line data (Turner 2007; Gil 2014), by named streets (Jiang and Claramunt 2004a, 2004b; Claramunt and Winter 2007; Jiang 2009), by axial lines (Penn et al. 1998; Karimi 2012), or axial graphs (Wagner 2008), or by route segments whose continuity is based on junction priority or the status of the road in a road hierarchy (Marshall 2005) (Figure 1).

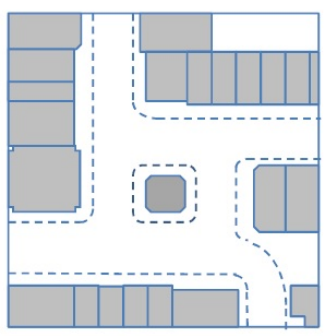

(a)

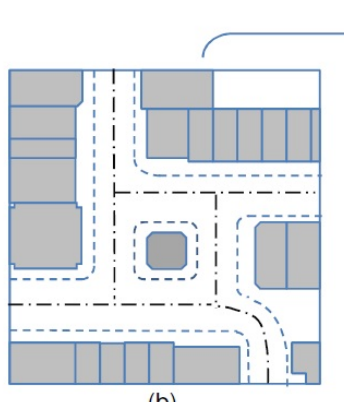

(b)

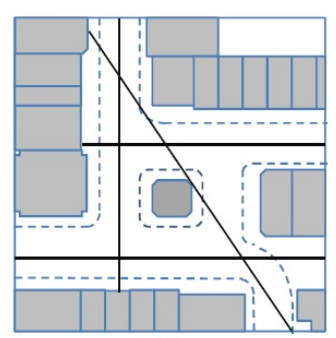

(c)

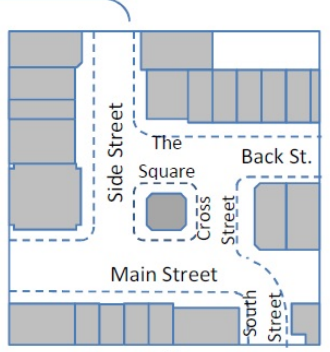

(d)

Figure 1: Alternative ways of selecting linear aspects of the environment for inclusion in a road network model. (a) 2-D map; (b) road centrelines; (c) axial lines; (d) named road sections. Each representation may generate a different structure in the network model.

1 This paper deals with "simple" undirected planar networks where each road has two distinct ends (no loops) and no multiple edges (between a given pair of vertices); furthermore, no road intersects with itself or with another road more than once. These conditions simplify the mathematical expression for demonstrative purposes herein, but the principles and analyses herein can readily be extended to other network conditions. 
Each of the alternative approaches (Figure $1 b, c, d$ ) has its own advantages and disadvantages (see, for example, Jiang and Claramunt 2002; Batty 2004, 2014; Marshall 2005; Porta, Crucitti, and Latora 2006b; Turner, Penn, and Hillier 2005; Turner 2007; Jiang and Okabe 2014). As with any representation (Lord and Wilson 1984), the choice will concern the purpose and context of application, including the availability and format of data. In principle, it seems beneficial that the representation should model elements-such as roads or their center-lines-that are actually used in the design and management of the road system. This paper is not further concerned with which aspects of the road system are selected to generate the network model. The main point has been to separate this issue conceptually from the following ones.

\subsection{Configurational representations}

\subsubsection{Elements for representation}

A graph $(G)$ is a set of elements and relationships (see, for example, Gross and Yellen 1999; Diestel 2000; West 2001; Wilson 2010) whose diagrammatic expression in the form of a set of points and lines (Deo 1974; Gross and Yellan 1999; Clark and Holton 1991) may be used to represent a wide variety of situations in which "points and connections between them have some physical or conceptual interpretation" (Gross and Yellen 1999).

Graphs have been applied to many fields including engineering, electronics, social sciences, operations research (see, for example, Barnes and Harary 1983; Foulds 1992). Of most direct relevance here, graph theory has found significant application to the analysis of transport networks where there is an intuitive and obvious relationship between the links and nodes in a transport network and the edges $(E)$ and vertices $(V)$ in a graph $G$ (for example, Kansky 1963; Morlok 1967; Bell and Iida 1997; Banks 1998; Buckwalter 2001; Barthélemy 2011). This conventional approach—sometimes referred to as a "primal" approach (Porta, Crucitti, and Latora 2006a) - has been used in several recent analyses of road networks (Buhl et al. 2006; Cardillo et al. 2006; Porta, Crucitti, and Latora 2006a; Lämmera, Gehlsena, and Helbinga 2006; Scellato et al. 2006; Masucci, Stanilov, and Batty 2009; Strano et al. 2012). However, there is also an alternative approach, which is to represent linear elements such as roads as the vertices in a graph, and the intersections as edges-the so-called "dual" graph.

Figure 2 shows diagrammatic representations of three transportation networks (a, b, c), each of which could be represented by the same primal graph $(G)$ (Figure $2 \mathrm{~d}$ ). However, for an air network, or ferry network (Figure 2a), it may be assumed that each link represents a point-to-point service; that is, there may be no continuity of services through nodes. For the rail network, some services continue through nodes (Figure 2b), while for the road network (Figure 2c), there is (in this case) a continuous road through each junction. This means that the structure of these three networks is different; however, when represented as a primal graph $(G)$ this difference is not captured. That is, it is not embodied in the structure of the graph, and cannot be directly inferred from the diagram (though it might be appended by labeling or other association, indirectly). In other words, while cases (a), (b), and (c) each map to (d), we cannot infer from (d) a unique correspondence with (a), (b), or (c). 


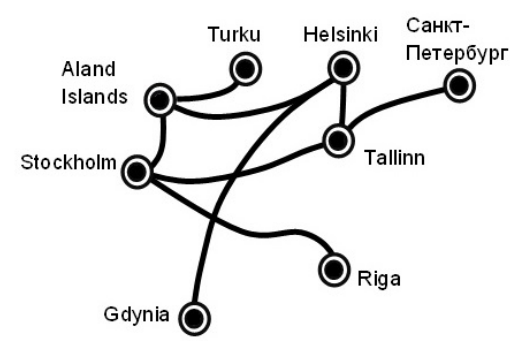

(a)

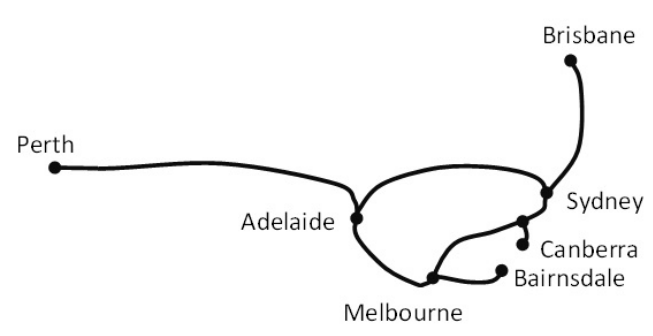

(b)

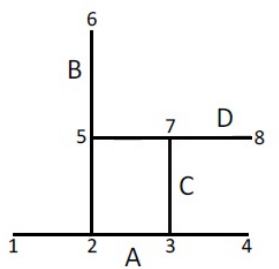

(c)

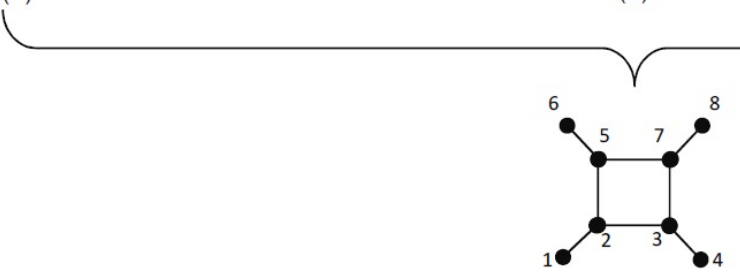

(d)

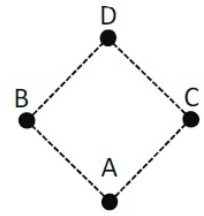

(e)

Figure 2: Alternative ways of representing transport networks: (a) Baltic ferry network diagram (selected routes); (b) Australian rail network diagram (selected routes); (c) a road network diagram, featuring 4 roads $($ A-D), or 8 links; (d) "Primal” graph $(G)$ corresponding to (a), (b) or (c); (e) "dual” graph $(G$ ") corresponding to road network (c).

To represent the continuity of roads through junctions, Figure 2 also shows how the road network (Figure 2c) could alternatively be represented as a dual graph $\left(G^{\prime \prime}\right)$ (Figure 2e). Before going further it is necessary to clarify terminology. Although the terms "primal" and "dual" have been used to describe the two kinds of graph representation shown in Figures $2 \mathrm{~d}$ and $2 \mathrm{e}$, this usage is not ideal. First, mathematically, a dual of a graph $G$ (denoted $G^{*}$ ) traditionally means there is a direct correspondence between one and the other (such that $\left(G^{*}\right)^{*}=G$ ); but we cannot get directly from Figure $2 \mathrm{~d}$ to e or vice versa. (As such, the term "dual" might better be reserved for $G^{*}$, which would refer to relationships between the spaces and buildings (etc.) in the interstices between the roads; see, for example, Courtat, Gloaguen, and Douady 2011). Second, the term "dual" may connote something secondary, derivative, or perhaps duplicative, but this seems unjustified, since both Figure $2 \mathrm{~d}$ and e can be obtained equally directly from $2 \mathrm{c}^{2}$ Nevertheless, the pair of terms "primal" and "dual" have the benefit of brevity and convey a ready sense of connection and contrast between one and the other; moreover, a number of recent papers on road network structure have adopted this terminology. In this paper, for present purposes, the "primal" and "dual" graph terms will be used following the convention of Porta, Crucitti, and Latora 2006a and 2006b, but will also be denoted for convenience as $G$ ' and $G$ ", which in the future could be used independently of any particular labeling in English.

Aside from terminology, the merits of using one kind of graph representation over another have been debated in the literature. Three reasons in favor of primal over dual representation are: (i) compatibility with

2 Alternative terms for "dual graph" have been suggested, such as "second-order topology" (Courtat, Gloaguen, and Douady 2011), but this also suggests that this graph is somehow further removed from the original network. In representing linear elements as vertices, the "dual" graph is similar to the "line graph," "interchange graph," or "edge graph" (http://mathworld.wolfram.com/LineGraph.html); however, in the case of the line graph, it is a direct transposition from the normal graph. This direct transposition does not apply with "primal" and "dual" graphs of Figures $2 d$ and e. 
established conventions across different fields (Batty and Rana 2004) (i.e., Figure $2 \mathrm{~d}$ relates c to a and b); (ii) the primal graph maintains the natural visual association - the linear elements on the ground are represented by linear elements in the graph (Batty 2004); while (iii) the dual "privileges" lines or streets as the focus of interest rather than locations or intersections (Batty 2004). Meanwhile, other authors have employed or put forward the case for the dual representation (Porta, Crucitti, and Latora 2006b; Jiang and Claramunt 2004a; Jiang 2007; Masucci, Stanilov, and Batty 2013). Which of these arguments applies or proves critical will vary according to context. But for now, let us consider in more detail some reasons for the importance of considering the dual approach for representation of road networks. These are: (1) focus on linear elements; (2) constitution of lines between intersections; (3) and continuity of lines through intersections.

\subsubsection{Focus on linear elements}

Graph theory applications allow understanding of elements of a given type through their relationships with other elements, where typically the vertices represent the elements, and the edges represent the relationships (Godehardt 1990) (Table 1). ${ }^{3}$ For example, the relation between cities (represented by vertices) can be understood by their road connections (represented by edges), which happen to be physically linear. As Erat, Löchl, and Axhausen (2008) note, "transport networks are embedded in real space where nodes and edges occupy precise positions in the three dimensional Euclidian space and edges are real physical connections." This physical linearity applies to roads (the primary focus of this paper), and also to river systems, tree branches and engineering structures.

Table 1: Elements and relationships suitable for graph representation (after Wilson and Beineke 1979; Clark and Holton 1991; Gross and Yellen 1999).

\begin{tabular}{lll}
\hline Context & Element (vertex) & Relationship (edge) \\
\hline Social networks & People & Social ties \\
Food chain & Organism & Consumption \\
Chemistry & Atom & Molecular bond \\
Sport & Team & Matches between teams \\
Linguistics & Word & Grammatical relationship \\
Activity-scheduling & Activity & Path dependency \\
Architecture & Rooms & Walls or doorways \\
Transportation & City & Transport link \\
\hline
\end{tabular}

However, just because an entity happens to be physically linear — whether a road, river, tree branch, or structural member-does not preclude its representation as a vertex in a graph, when that linear element is the primary focus of attention. In the case of road networks, the individual roads (though linear) can be represented as vertices, while their connections (i.e. junctions) can be represented as edges. This "dual" approach seems justified when the roads themselves are indeed the primary focus of attention.

\subsubsection{Constitution of lines between intersections}

Graph representations - with their categorical distinctions between vertices and edges-make sense because the vertices and edges typically relate to categorically different kinds of things: in a social network, people are categorically distinct from social ties; in sports, teams are categorically distinct from fixtures. Moreo-

${ }^{3}$ In many cases, the element is in a sense primary (i.e., a first order object) because the elements can exist without relation-

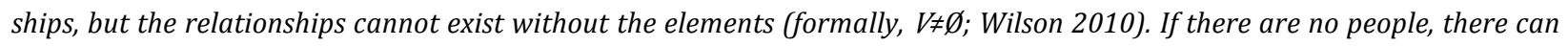
be no social relationships; if there are no atoms, there can be no molecular bonds. 
ver, in a graph there is nothing existing "between" two elements but the relationship itself. (There are no persons "halfway along" a social tie.) What comprise vertices and edges are mutually exclusive (i.e., $V \cap E=\varnothing$; Diestel 2000).

But for a road network, at the level of resolution considered here (i.e., where nodes represent junctions rather than whole cites), there is not such a categorical difference between what happens along a road and what happens at the ends of the roads or their intersections. ${ }^{4}$ So while it may be visually intuitive to represent linear roads by linear links in a graph, it is not necessarily intuitive or optimal to divide the elements of the road network into two sets as shown in Figure 3; this invites the possibility for alternative representations.

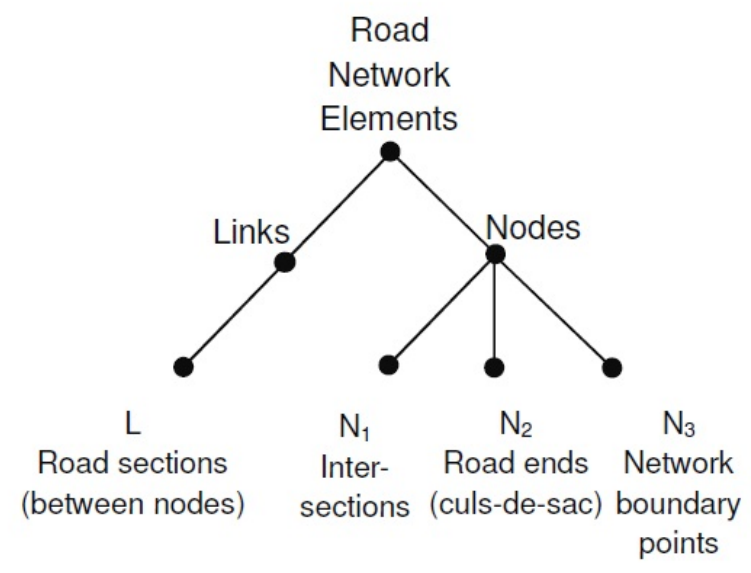

Figure 3: Deconstruction of the primal graph representation $(G)$. The three kinds of road network element represented by nodes $\left(\mathrm{N}_{1}, \mathrm{~N}_{2}, \mathrm{~N}_{3}\right)$ have no more in common with each other than they have with the elements represented by links (L); hence the classification of elements implicit in the 'primal' representation is in a way artificial.

\subsubsection{Continuity through intersections}

Topology and graphs are often associated in network analysis, but we can recognize a difference in emphasis between the two: In graph theory, the emphasis tends to be on the connectivity between discrete entities (Hayes 2000), whereas topology is centrally concerned with continuity of entities (Bredon 1997). In the context of road network representation, the question becomes: Which aspect is more significant-the continuity of a road, or its decomposition into discrete segments? For example, consider a situation where a main route goes through a town from one end to the other and has several side roads (for example, Figure 1, Figure 2c). Is it more important that we recognize the main route (A) as a single continuous entity, with several roads off it, or that we recognize a set of eight individual road segments, some of which may happen to join points (1-4) "in series"? (This is a question typically overlooked in those studies that go straight to the primal graph). The graphs ( $G$ and $\left.G^{\prime \prime}\right)$ in Figures 2 (d) and (e) show different interpretations of the same network. One representation is not intrinsically better than the other. The value of either will depend on the particular purpose and context of application. Here, where the structure of a network is concerned (as opposed to, say, spatial central-

${ }^{4}$ For urban streets, the distinction is particularly blurred, because a street is not just a conduit between urban destinations but can be a destination in its own right. Indeed, a section of street frontage between intersections is more likely to be a destination than an intersection is. 
ity of locations), it seems that the relation between main and subsidiary elements must be intrinsically worth considering.

\subsubsection{Direct representation of continuity plus segmentation}

There is a variety of existing approaches to road network representation and analysis that directly represent roads as continuous entities - may be continuous through junctions - and not broken into discrete segments that (only) span between junctions (e.g. Thomson and Richardson 1999; Turner 2007; Jiang 2007; Tomko, Winter, and Claramunt 2008). Of particular interest here is the route structure analysis approach (Marshall 2005) because it combines the recognition of the continuity and segmentation of routes. The small network in Figure $2 \mathrm{c}$ can be interpreted as a route structure. It simultaneously embodies a set of four routes (elements that are continuous through junctions) and eight links (segments). Route structure analysis uses as its basic element the route, which may be derived from a road layout according to a number of criteria, which could include named streets and continuity of physical alignment, as used in other nominally distinct approaches. What is important here is that the analytic part of route structure analysis uses elements that are continuous through junctions - howsoever that continuity may be obtained or defined-while also taking account of segmentation. Although route structure analysis has not been widely applied in road network analysis, it is of interest here because it explicitly considers the continuity of routes through junctions, both in terms of visual representation and analysis of properties such as "continuity," and how this relates to the hierarchical structure of road networks.

Route structure analysis may be perceived to be distinct or even somehow removed from conventional primal $\left(G^{\prime}\right)$ and dual $\left(G^{\prime}\right)$ graph approaches; and yet, the question arises as to how it might be linked to these. In fact, other forms of network representation—such as axial maps and line segments—also use some kind of line that is continuous through intersections to represent roads that are continuous through junctions. This paper therefore considers what kind of mathematical entity might underpin these forms of representation, which shall be identified herein as a line structure. The properties of this mathematical object are discussed in the next section (3), and their relation to $G$ and $G$ ' is discussed in Section 4.

\section{$3 \quad$ Line structure}

A point is that of which there is no part. And a line is a length without breadth. And the extremities of a line are points. -Euclid, Elements. ${ }^{5}$

\subsection{Basics}

Let us use the term line structure to denote a "topological" structure made up of lines. Here, lines connote abstract mathematical entities representing real-world features that are linear, such as roads. Structure connotes that we are dealing with relationships between connected sets of elements. So a line structure could be used to represent a network (connected set) of roads. Topological means that we are only interested in certain topological properties of the structure, such as order and incidence, continuity and connectivity. In contrast to Euclidean geometry, we omit consideration of the metric length of the line, its orientation, and whether it

${ }^{5}$ Euclid's Elements has been described as the most influential textbook-not just in geometry but also in the history of civilization (Faber 1983). This particular translation (Fitzpatrick 2008, p. 6) is used to emphasize the continuity between the first three axioms. Euclid's fourth axiom refers to a straight line; this straightness takes us into elements of geometry that lie beyond the scope of line structure. 
is curved or straight. But what is retained is the sense that a line is constituted by points at and between extremities and intersections, and that a line may terminate on another line, or may continue through an intersection (Figure 4).

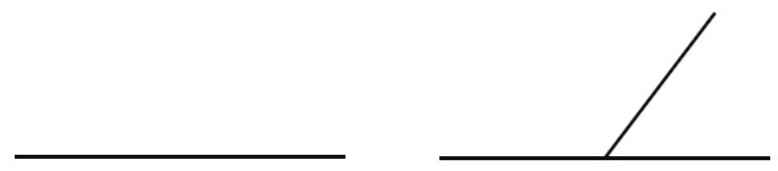

(a) (b)

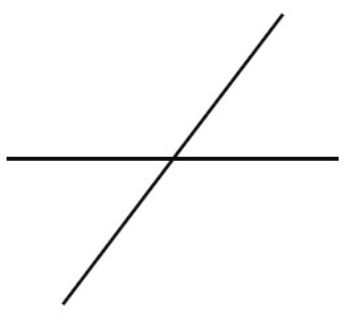

(c)

Figure 4: Line structure elements retained from Euclidean geometry (after Mackay 1893, 1). (a) A line, having two ends, each of which is a point. (b) Two lines connecting, one continuous through the point of intersection. (c) Two lines intersecting; both continuous.

Indeed we have already seen this kind of structure, unremarked, earlier in the paper. The diagram depicted in Figure 2 (c) could be interpreted as a line structure. In fact, this kind of diagram crops up from time to time elsewhere in the literature (for example, Bejan 1996; Marshall 2005; Masucci et al. 2009) but is typically unremarked in terms of the kind of representation it constitutes.

A line structure may be drawn so that each straight line is considered a single entity (Figure 5). In Figure 5 , line $\mathrm{A}$ is continuous through its intersection with line $\mathrm{C}$, while line $\mathrm{B}$ is continuous through its intersection with lines D and E. Elsewhere, where road sections may be physically collinear (e.g., through a junction), but have separate identities, this can be shown visually by artificially deflecting the lines or by labeling. Otherwise lines may be depicted visually as curved to allow changes of orientation while indicating continuity. Where a line is broken, i.e. has an angular (not curved) change in direction, this is considered more than one line, unless indicated otherwise. For example, in Figure 5, A and B meeting at 90 degrees are two separate lines, and $\mathrm{D}$ and $\mathrm{E}$ meeting at an acute angle are separate lines. (Straightness of lines in Figure 4 and 5 is used solely to visually indicate continuity; the lines could be curved as long as it is clear where lines are continuous.)

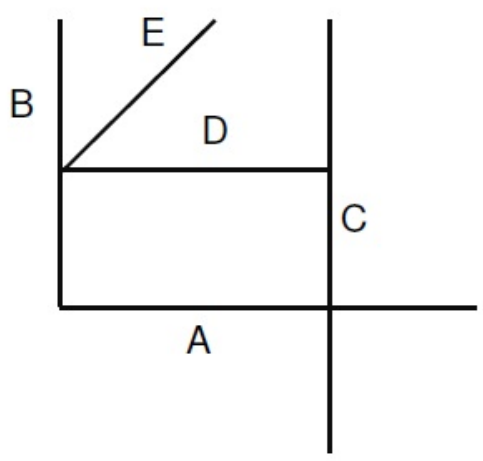

Figure 5: A line structure, $S^{\#}$, comprising 5 lines $\{A, B, C, D, E\}$ 
In fact, it is possible to interpret a route structure, axial map, or any connected set of lines that may be continuous through intersections (e.g., Bejan 1996; Masucci et al. 2009), as a line structure. The difference (or equivalence) between a line structure and a route structure (or axial map, etc.) is analogous to the difference (or equivalence) between a graph and a conventional "link and node" network diagram in the following way.

A conventional road network diagram is an analytic or representational device, comprising nodes (or junctions) and links used in the road network context. It can be interpreted mathematically as a graph. A graph itself is an abstract mathematical entity, comprising sets of elements (vertices and edges). A graph need not represent a network or take the form of a diagram.

A route structure is an analytic or representational device, comprising routes and junctions or joints, used in the road network context. Similarly, an axial map is an analytic or representational device, comprising axial lines and their intersections, used in the context of road networks or other spatial configurations. Both route structures and axial maps can be interpreted as line structures. A line structure itself is an abstract mathematical entity (a linearly ordered incidence structure) comprising sets of elements (lines and their intersections). A line structure need not represent a network or take the form of a diagram.

The question becomes how can we define such a kind of line structure mathematically, and what are the consequent properties.

\subsection{Line structures as incidence structures}

A full formal mathematical account of the definition and properties of line structures would take us into realms of abstract mathematics (e.g., in relation to various kinds of geometry, finite spaces or line spaces; e.g., Lord and Wilson 1984; Buekenhout and Cohen 2013) that do not directly concern road network analysis. For present purposes it shall suffice to interpret a line structure as a kind of linearly ordered incidence structure. An incidence structure $S$ may take the form of a triple $(X, Y, I)$ where $X$ is a set of lines, $Y$ a set of points and $I$ the set of incidence relations between $X$ and $Y$ (see, for example, Buekenhout 1995, Rosen 2000). Conventionally, the elements of any $X$ and $Y$ are not ordered; hence if $X=\left\{x_{i}, x_{j}, x_{k}\right\}$, then $i, j$ and $k$ are in no particular order, and $\left\{x_{i}, x_{j}, x_{k}\right\} \equiv\left\{x_{i}, x_{k}, x_{j}\right\}$, etc.

We can then recognize a line structure $\left(S^{\#}\right)$ as a special kind of incidence structure $(L, P, I)$ in which the elements (i.e., points) comprising each line $(L)$ are linearly ordered: for any line $L, i<j<k$, hence $\left\{x_{i}, x_{j}, x_{k}\right\} \neq\left\{x_{i}, x_{k}\right.$, $\left.x_{j}\right\}$. Moreover, points lie on lines; or put another way, the incidence relations between lines are points. This means that to specify $S^{\#}$, we only need to specify two components $(L, I)$ or $(L, P)$. Such an incidence structure can be specified by some sort of incidence matrix (see next section).

In fact, an incidence structure (depending on exactly how defined) could be seen as a generalization of a graph (e.g., Lord and Wilson 1984) — a more general kind of structure, against which a graph is but a specialized (limited) kind of incidence structure in which certain elements (edges) connect only pairs of other elements (vertices). In an incidence structure, elements may contain any number of other elements. Hence, put simply, in a line structure, a line may go through any number of points. This allows it, intrinsically, to represent continuity through points of intersection in a way that a conventional graph (directly, and visually) necessarily cannot.

\subsection{Line structure specification by incidence matrices}

In fact, we can identify two different variants of line structure: one continuous, the other discrete. These will be referred to as the parametric line structure and the ordinal line structure. These are graphically equivalent - that is, when drawn on the page they have the same structure of lines-but are mathematically distinguishable in that in the parametric line structure, lines are constituted by a continuum of points (as in Euclid- 
ean geometry), whereas in the ordinal line structure, the only points discretely defined are those such as intersections or pendant ends. Either way (and in common with graphs), these line structures can be considered as sets of elements and relationships, even without their being represented diagrammatically as lines.

\subsubsection{Parametric line structure}

In a parametric line structure $S_{\mathrm{P}}^{\#}$, each line is a linear continuum of points, as in Euclidean geometry. Hence to define $S_{\mathrm{P}}^{\#}$, we need to specify the end points and intersection points of each line. In Cartesian (coordinate) geometry, a line may be represented in terms of $x$ and $y$ coordinates, or in terms of some other parameter (via parametric equations). Here, we specify the lines in terms of parameters, such that a line is a linearly ordered set of points on a given interval. We can apply the following conventions:

1. Each line $X_{i}$ has a parameter $x_{i}$ indicating position along the line, equivalent to the abscissa ( $x$-value) along the $x$-axis in co-ordinate geometry. Here, however, there is no Cartesian plane, just a set of lines, each of which is its own "axis." In Figure 6, line A has a parameter $a$, and line B has parameter $b$, and so on.

2. Let this parameter $x_{i}$ be a real number, being 0 at one end of the line and 1 at the other. Hence any line $X_{i}=\left\{x_{i} \in \mathrm{R} \mid 0 \leq x_{i} \leq 1\right\}$. By this convention, we can represent the fact that a line could in principle extend below parametric value 0 or exceed 1, but that only the line segment between 0 and 1 inclusive is part of the network under scrutiny. Note that the values of the parameters here may be flexibly allocated. For example, the intersection points along line $\mathrm{C}$ are given here as $c=1 / 3$ and $c=2 / 3$ but those could be any fractional values as long as they are in the correct numerical order (e.g, they could be $c=0.1$ and $c=0.2$, etc). ${ }^{6}$

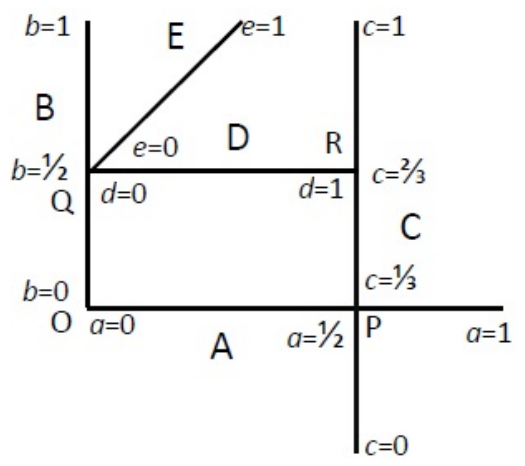

Figure 6: A parametric line structure, $S_{\mathrm{P}}^{\#}$

Any point on a line $X$ can be specified by the value of parameter $x$; therefore, the set of points $(P)$ in $S_{\mathrm{P}}{ }^{\#}$ is "internalized" in the parametric definitions of the lines $(L)$. Furthermore, an intersection point can be specified by the two (or more) lines intersecting $(P=I \subseteq L \times L)$. Any point can be expressed as a combination of the parametric values of any or every line in the set. So a point $Y$ can be expressed as $Y\left(x_{1 Y}, x_{2 Y}, \ldots, x_{n Y}\right)$, where $x_{i Y}$ is the parametric value of the point $Y$ on line $x_{i}$.

In Figure 6 , let $\mathrm{O}$ be the point $(0,0,-,-,-)$ where $a=1, b=0$ and the dash $(-)$ indicates that $\mathrm{O}$ does not lie on lines $\mathrm{C}, \mathrm{D}$ or $\mathrm{E}$. Let $\mathrm{P}$ be the point $(1 / 2,-, 1 / 3,-,-)$, and so on. Hence, using this "coordinate topology," all intersection points can be specified. A parametric line structure $S_{\mathrm{P}}^{\#}$ can hence therefore be specified as follows: $S_{\mathrm{P}}^{\#}=(L, P) ; L=\left\{X_{1}, X_{2}, X_{3}, \ldots X_{n}\right\} ; X_{n}=\left\{x_{i} \in \mathbf{R} \mid 0 \leq x_{i} \leq 1\right\}$ for each of $n$ lines; $P=\left\{Y_{1}, Y_{2}, Y_{3}, \ldots Y_{m}\right\} ; Y_{m}\left(x_{1 Y}, x_{2}, \ldots\right.$, $\left.x_{m \mathrm{Y}}\right)$ for $m$ intersections.

In Figure 6, the line structure $S_{\mathrm{P}}^{\#}$ is given by: $S_{\mathrm{P}}^{\#}=(L, P) ; L=\{A, B, C, D, E\} ; A=\{a \in \mathbf{R} \mid 0 \leq a \leq 1\} ; B=\{b \in \mathbf{R} \mid$ $0 \leq b \leq 1\} ; C=\{c \in \mathrm{R} \mid 0 \leq c \leq 1\} ; D=\{d \in \mathrm{R} \mid 0 \leq d \leq 1\} ; E=\{e \in \mathrm{R} \mid 0 \leq e \leq 1\} ; P=\{\mathrm{O}, \mathrm{P}, \mathrm{Q}, \mathrm{R}\}$; and

${ }^{6}$ Alternatively, these could be specified proportionately in relation to metric distance. However, this possibility is not pursued further in this paper. 


$$
\begin{aligned}
& \mathrm{O}(0,0,-,-,-) \\
& \mathrm{P}(1 / 2,-, 1 / 3,-,-) \\
& \mathrm{Q}(-, 1 / 2,-, 0,0) \\
& \mathrm{R}(-,-, 2 / 3,1,-)
\end{aligned}
$$

The line-ends, and all intermediate points between intersections, are therefore included implicitly (e.g., the existence of a point at $a=1$ is inferred, even if not explicitly specified). The set of points ("topological coordinates") can be represented as an $m \times n$ incidence matrix, where $m$ is the number of intersection points $(m=|P|)$ and $n$ is the number of lines $(n=|L|)$. This can be referred to as the parametric point matrix, with the general form:

$$
\left(\begin{array}{cccc}
x_{1}\left(Y_{1}\right) & x_{2}\left(Y_{1}\right) & \ldots & x_{n}\left(Y_{1}\right) \\
x_{1}\left(Y_{2}\right) & x_{2}\left(Y_{2}\right) & \ldots & x_{n}\left(Y_{2}\right) \\
\ldots & \ldots & & \ldots \\
x_{1}\left(Y_{m}\right) & x_{2}\left(Y_{m}\right) & \ldots & x_{n}\left(Y_{m}\right)
\end{array}\right)
$$

In the parametric point matrix, each value $x_{i}\left(Y_{j}\right)$ means the parametric value $x_{i}$ along line $X_{i}$ at the point $Y_{j}$. Each value in a given column must be unique (else two points would be coincident). Each column can have at most one entry with a value of 0 , and at most one entry with a value of 1 (indicating the end points of the associated line); it may have any number of distinct intermediate values between 0 and 1 . For the network in Figure 6 this gives:

$$
\mathrm{O}\left(\begin{array}{ccccc}
a & b & c & d & e \\
\mathrm{P} & 0 & - & - & - \\
\mathrm{Q} \\
\mathrm{R} \\
1 / 2 & - & 1 / 3 & - & - \\
- & 1 / 2 & - & 0 & 0 \\
- & - & 2 / 3 & 1 & -
\end{array}\right)
$$

In this case, each row in the matrix gives the topological (parametric) coordinates of each point (see definitions of points $\mathrm{O}, \mathrm{P}, \mathrm{Q}, \mathrm{R}$ earlier). Each column gives the intersection points along a line, so for example, reading down column $c$ tells us that line $\mathrm{C}$ has intersection points at $\mathrm{P}($ at $c=1 / 3$ ) and $\mathrm{R}$ (at $c=2 / 3$ ) (Figure 6).

The parametric line structure may also be expressed in the form of a parametric line matrix. Here, we have an $n \times n$ matrix, where $n$ is the number of lines $(n=|L|)$, and where each value $x_{i}\left(X_{j}\right)$ indicates an incidence relation $I$, where $I \subseteq L \times L$. Specifically, each value $x_{i}\left(X_{j}\right)$ means the parametric value $x_{i}$ along line $X_{i}$ at its point of intersection with line $X_{j}(i \neq j)$ :

$$
\left(\begin{array}{cccc}
- & x_{2}\left(\mathrm{X}_{1}\right) & \ldots & x_{n}\left(\mathrm{X}_{1}\right) \\
x_{1}\left(\mathrm{X}_{2}\right) & - & \ldots & x_{n}\left(\mathrm{X}_{2}\right) \\
\ldots & \ldots & & \ldots \\
x_{1}\left(\mathrm{X}_{n}\right) & x_{2}\left(\mathrm{X}_{n}\right) & \ldots & -
\end{array}\right)
$$

For the line structure in Figure 6, we get:

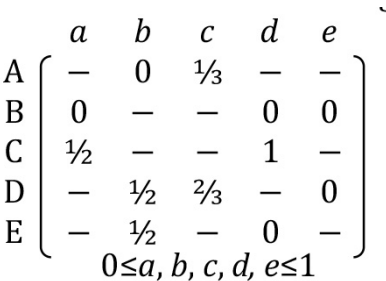


This line matrix tells us, for example, that (reading across row $\mathrm{A}$ ) line $\mathrm{A}$ intersects line $\mathrm{B}$ at $b=0$ and line $\mathrm{C}$ at $c=1 / 3$; or that (reading down column $a$ ) line A intersects line $\mathrm{B}$ at $a=0$ and line $\mathrm{C}$ at $a=1 / 2$. Note that the positions of numerical (non-dash) values are symmetrical about the primary diagonal. As with the point matrix, the line matrix - together with the specification of the range values of $a, b, c, d$, e-gives a complete specification for the line structure. (This implicitly includes all positions along all the lines, including, for example, points at $c=1 / 2$ or $d=1 / 4$, etc.)

Overall it can be understood that a parametric line structure is a topological structure one step removed from linear Euclidean geometry: A parametric line structure does not have absolute location, orientation, metric length or curvature, but lines are composed of a linearly ordered set of points, which can indicate positional and structural information.

In terms of representation of the real world, the parametric line structure $\left(S_{\mathrm{P}}^{\#}\right)$ - unlike the graph—can have lines with any number of points along them representing the continuity of roads with an indefinite number of points along them (whether intersecting or otherwise). In other words, line structure is not simply a matter of visual presentation of a structure but is about the fundamental fit of a mathematically continuous entity (line) with a physically continuous entity (road) on the ground.

\subsubsection{Ordinal line structure}

We can also recognize an ordinal line structure $S_{\mathrm{O}}{ }^{*}$ where the lines comprise only discrete end-points and intersection points but no "intermediate" points as in $S_{\mathrm{P}}^{\#}$. Indeed we can recognize an ordinal structure as a structure (intersecting set) of linearly ordered discrete sets, where each linearly ordered set can be drawn as lines (as with a graph) and hence used to represent a road network. The ordered sets could be sets of numbers, or letters, or any other set with a definite order of distinct (non-recurring) elements.

Let $S_{\mathrm{O}}{ }^{\#}$ be the set of lines $L$ and points $P$; where $L=\left\{X_{1}, X_{2}, \ldots X_{3}\right\}$ and where any line $X_{i}$ comprises a linearly ordered set of $n$ elements, $\left\{x_{1}, x_{2}, \ldots, x_{n}\right\}$, being the linearly ordered set of discretely identified points along the line. Since order matters, $\left\{x_{i}, x_{j}, x_{k}\right\} \neq\left\{x_{i}, x_{k}, x_{j}\right\}$. In Figure $7, L=\{A, B, C, D, E\} ; P=\{1,2,3,4,5,6$, $7,8,9\} ; A=\{1,2,3\} ; B=\{1,4,5\} ; C=\{6,2,7,8\} ; D=\{4,7\} ; E=\{4,9\}$. This set of information completely specifies $S_{\mathrm{O}}^{\#}$.

The ordinal line structure has the same graphic profile as the parametric one, although (where appropriate) we could distinguish the two by using a dashed line for $S_{\mathrm{O}}$ " (as in Figure 7) to indicate that there is "nothing" between the points. Compared with the parametric line structure, the ordinal structure loses the continuum of points between intersections and end points but gains explicit naming of end and intersection points as part of the specification. Either way, the integrity of the lines is maintained and their continuity through intersections. 


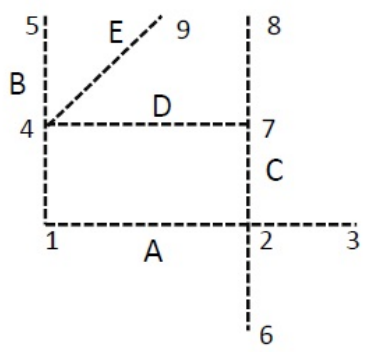

Figure 7: An ordinal line structure $S_{\mathrm{O}}^{\#}$, corresponding with Figures 5 and 6

An ordinal structure $S_{\mathrm{O}}{ }^{\#}$ can be specified in the form of an ordinal point matrix $(L \times P)$. Here, rather than parametric values, the entries are ordered points along the line, e.g. $A_{1}, A_{2}, A_{3}$ (or numerical labels 1.1, 1.2, etc.). Unlike $S_{\mathrm{P}}{ }^{\#}$, we need to specify the existence of pendant ends, since they cannot necessarily be inferred:

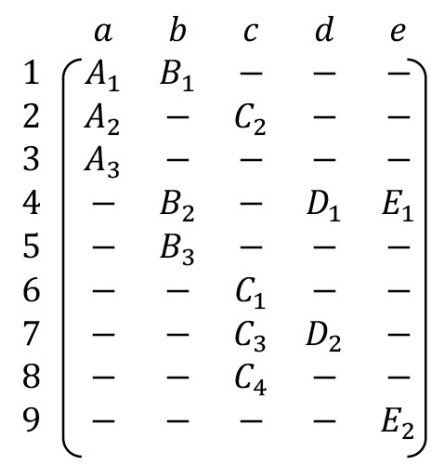

We can also specify an ordinal line matrix, where the positions are $\mathrm{X}_{\mathrm{i}}$ along each line:

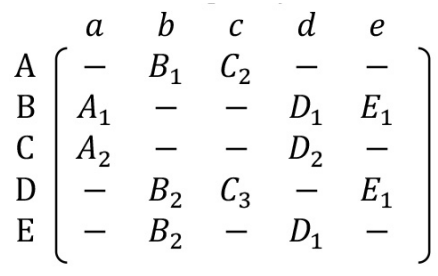

From this line matrix we can infer coincident points-these being symmetrical across the primary diagonal, such as $A_{1}$ corresponding to $B_{1}$ (also, we can infer $B_{2}=D_{1}=E_{1}$ ). However, this does not yet give a complete specification of the structure; for a complete specification, we would also need to separately specify the set of pendant ends - in this case, to thereby include $\mathrm{A}_{3}, \mathrm{~B}_{3}, \mathrm{C}_{1}, \mathrm{C}_{4}$ and $\mathrm{E}_{2}$-for all lines.

As a set of elements and relationships, an ordinal structure (like incidence structures in general) can be seen as a more general form of a graph, where elements (points) are not just related in pairs, but in strings of any number $(n \in \mathrm{N})$ of linearly ordered elements. Conversely, a graph can be seen as a special kind of ordinal structure in which all the linear sets have only two elements $(n=2)$. (In the incidence matrix for a graph, equivalent to the ordinal point matrix $(L \times P)$, each column would have exactly two entries, as in the case of 
column $d$ or $e$ in the ordinal point matrix above.) In moving from an ordinal structure $S_{\mathrm{O}}{ }^{\#}$ to a primal graph $G$, the lines are broken into individual line segments, where each line segment joins two nodes. In effect, a line $L\{1,2,3,4\}$ becomes three links, $\{1,2\},\{2,3\}$ and $\{3,4\}$. The graph $G$ is an extreme case where every line (linearly ordered set) that could be further decomposed into individual line segments (element pairs) is so decomposed. This invites further scrutiny about how the line structure and graph formats relate to each other.

\section{$4 \quad$ Relation between line structure and other representations}

\subsection{Line structure versus graph representations}

Figure 8 (a) shows a sketch of an arboreal tree, with a trunk and four branches. Below this, Figure 8 (d) shows a line-structure representation of the tree. From this line structure, we can tell several things about the realworld object that it represents: that it has a trunk (A) that has three branches off it $(B, C, D)$, and that the first branch up (B) itself has a branch off it (E). 


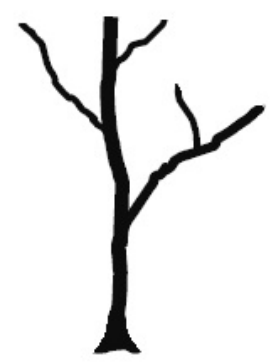

(a)

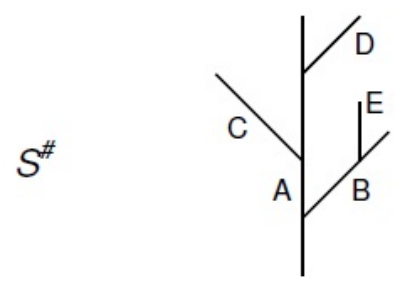

(d)

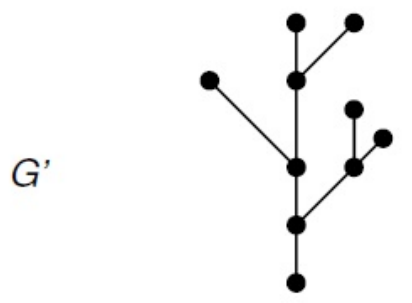

(g)

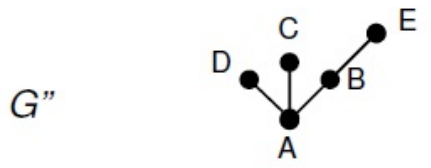

(j)

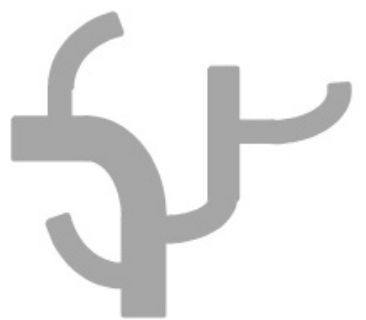

(b)

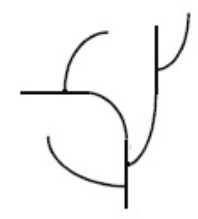

(e)

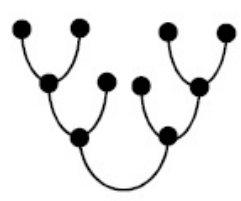

(h)

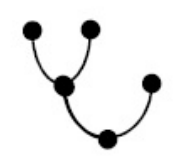

(k)

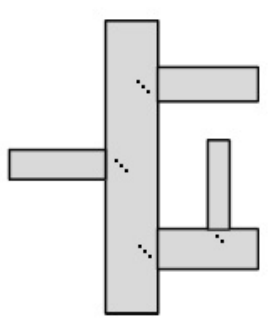

(c)

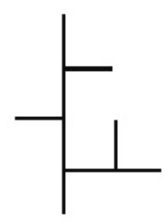

(f)

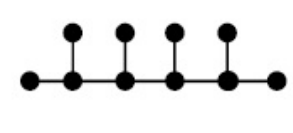

(i)

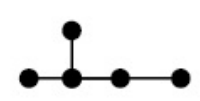

(l)

Figure 8: Representations of tree structures. Real-world entities: (a) tree; (b) road layout; (c) engineering structure. Line structures (d), (e) and (f) are equivalent to each other, and represent any of (a), (b) or (c). Primal graphs (g), (h) and (i) are equivalent to each other, and also correspond to (d), (e) or (f). Dual graphs (j), (k) and (l) are equivalent to each other, and also correspond to (d), (e) and (f) - but not directly to (g), (h) or (i).

The trunk is the longest element, comprising four line segments. The first branch up comprises two line segments while the remaining branches comprise one line segment each.

The same line structure (Figure $8 \mathrm{~d}$ ) could also be used to represent the road layout in Figure 8 (b) with its through (trunk) road and four side roads or the engineering structure in Figure 8 (c) comprised of a central column and four cantilevers. Alternatively, curved (Figure $8 \mathrm{e}$ ) or orthogonal (Figure $8 \mathrm{f}$ ) variants of the line structure representation could be used. In each case, the same five linear elements are identifiable, and the same relations between primary and subsidiary elements are fixed; for our purposes Figure 8 (d), (e) and (f) 
are topologically equivalent line structures, and could be used to represent any of the entities in Figure 8 (a), (b), and (c).

Now consider the third row (Figure $8 \mathrm{~g}-\mathrm{i}$ ). Here, the tree structure is represented as a primal graph in three equivalent variants. In moving from the second to third row, the graph loses the continuity of the trunk — and indeed the identity of the trunk as a single coherent entity (A) — and the hierarchical distinction between trunk, branch, and branch-off-branch. In other words, while the graph maintains the "configurational" (acyclic) sense of tree structure, it loses the "constitutional" (hierarchical, trunk-and-branch) sense of tree structure.

Next consider the fourth row, representing equivalent dual graphs (Figure $8 \mathrm{j}-1$ ). Here, the trunk and discrete branches retain their identities as elements $(\mathrm{A}-\mathrm{E})$, but the representation does not distinguish between them: One could not be certain which vertex represented the trunk and which the branches.

Graph theory is infused with arboreal metaphors: trees, leaves, forests, arborescences, and even arboretums. But a graph cannot intrinsically distinguish a trunk from a branch. In effect, the graph format is so flexible-it can represent so many different kinds of thing-that we lose something real about structure. Meanwhile, some artificial features are added. What is artificial is the conflation of referents under the artificial concept of the node: For a tree, a node can variously represent (i) the base of the trunk, (ii) the tips of the twigs, or (iii) joints between branches - as if node (i) were more like node (ii) or (iii) than the wood lying between (i) and (ii) and (iii). ${ }^{7}$ These losses and artificial additions may or may not be practically significant; the significance will depend on the purpose and context of application and must be taken into consideration when creating particular network models for analysis.

What is of concern here is the fundamental theoretical nature of these mathematical objects and their necessary attributes and relationships. In fact, it is possible to demonstrate the relations between line structures and graphs of different kinds in a systematic way. In the remainder of this section, we consider the relations between the line structure $S^{\#}$, the primal graph $G$, and the dual graph $G^{\prime \prime}$.

\subsection{Relations between $S^{\#}, G^{\prime}$, and G"}

It can be shown that the primal $\left(G^{\prime}\right)$ and dual $\left(G^{\prime}\right)$ graphs have no elements in common: $G^{\prime} \cap G^{\prime \prime}=\varnothing$ (see Table 2). In effect, the lines in the line structure $S^{\#}$ either become broken into individual links in $G$, or retained as whole entities represented by vertices in $G^{\prime \prime}$. Meanwhile, the interesctions in $S^{\#}$ either become vertices in $G^{\prime}$ or edges in $G^{\prime \prime}$. While the vertices on $G^{\prime}$ have corresponding points on the line structure, and while the links on $G$ constitute the same line segments on the line structure, these have different identities. ${ }^{8}$ Although $G^{\prime}$ and $G^{\prime \prime}$ are complementary, their union $G^{\prime} \cup G^{\prime \prime}$ is not enough to specify $S^{\#}$, because neither $G^{\prime}$ nor $G$ " can tell us the "continuity and termination conditions" (CTC): how many line segments each line is constituted by, or whether line $\mathrm{X}$ terminates upon line $\mathrm{Y}$, or vice versa. This invites consideration of what is this "missing" information.

\footnotetext{
7 In the line structure (Figure $8 d$ ), the positions of these nodal points-the base of the trunk, the tips of the twigs and the joints between branches-are of course present, and indicated by the ends of the lines, but they are not explicitly highlighted as categorically different from the wood between the joints. They are as alike or unalike as a point that is at the end of a line, or the point that is the intersection of lines, or a point midway along a line (cf. Figure 3).

${ }^{8}$ This identity disjunction has common-sense significance. Let R be the set of Roman Roads in Britain \{Watling Street, Ermine Street, Via Devana...\} and $A$ be the set of ' $A$ ' roads $\{A 1, A 2, A 3, \ldots\}$. A given stretch of road (say, Edgware Road in London) could coincide with both Watling Street and A5, but A5 is not an element of $R$ and no analysis of the set of $R$, of itself, will yield information about the set of $A$ roads, even if they contain stretches of actual road in common.
} 
Table 2: Relations between $S^{*}, G^{\prime}, G^{\prime \prime}$ and $S^{=}$

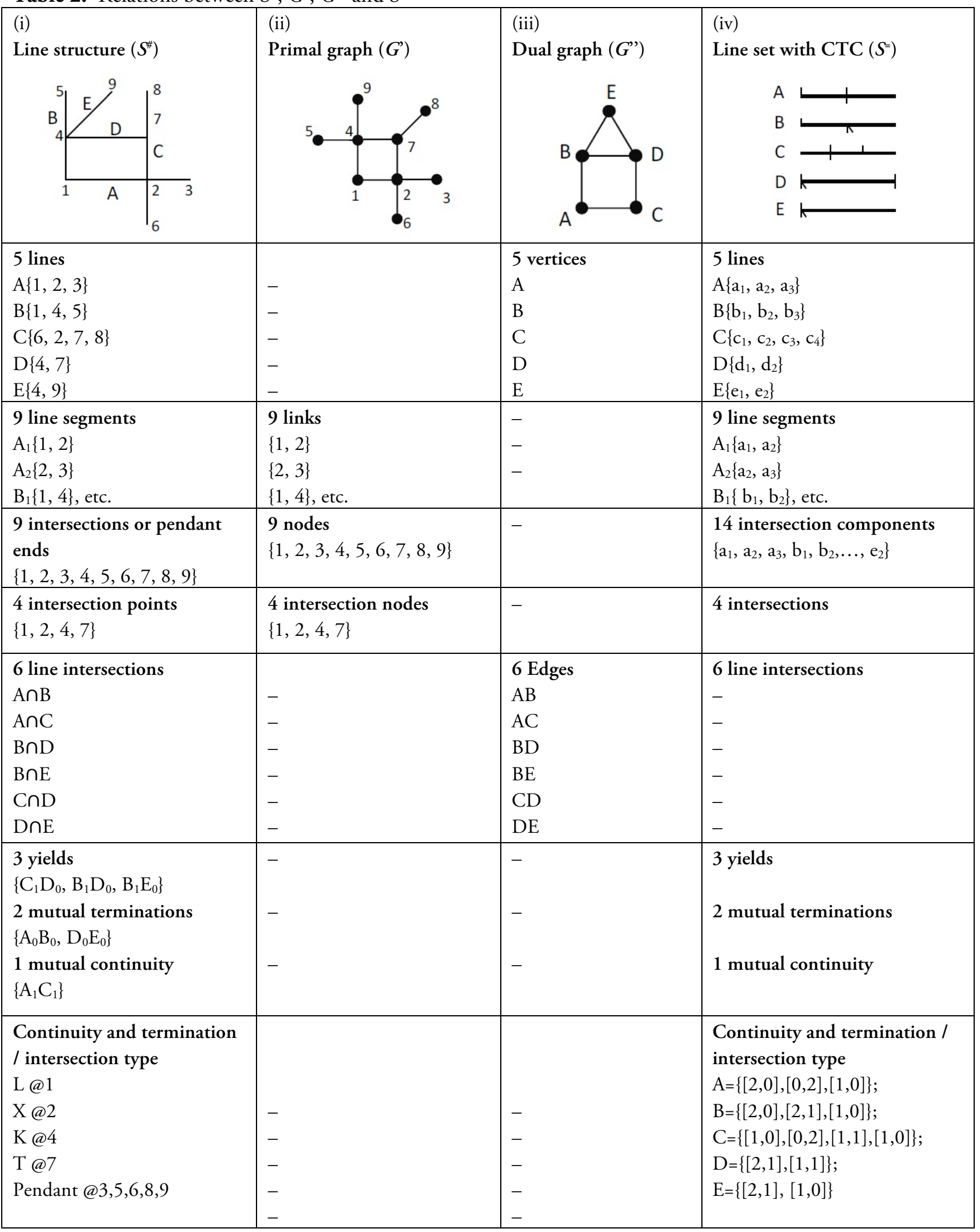




\subsection{Continuity and termination information: $S^{=}$}

We can define a "line set" $S^{=}$as the set of individual lines making up a line structure, together with their local continuity and termination conditions (here, the superscript $=$ denotes the lines considered individually), but not including information about which particular lines they connect to or what happens to those other lines beyond their intersection. By defining $S^{=}$this way, we aim to capture information in $S^{\#}$ that is complementary to $G^{\prime} \cup G^{\prime \prime}$; that is, information over and above what is contained in $G^{\prime}$ or $G^{\prime \prime}$ but without specifying the full structure $S^{\#}$ (which would happen if we defined all the intersection points, pendant ends and identity of lines in relation to each other).

Let $S^{=}$comprise the set of lines $L\left\{\mathrm{X}_{1}, \mathrm{X}_{2}, \ldots, \mathrm{X}_{n}\right\}$ plus the set of continuity and termination conditions, say $K\left\{\mathrm{~K}_{1}, \mathrm{~K}_{2}, \ldots, \mathrm{K}_{\mathrm{n}}\right\}$ corresponding to the set of lines. In general, for a line, with $n$ points (intersections or pendant ends; $n \geq 2)$, the continuity and termination conditions may be given by $\left\{\left[l_{01}, l_{11}\right],\left[l_{02}, l_{12}\right], \ldots,\left[l_{0 \text { n }}\right.\right.$, $\left.l_{1 \mathrm{n}}\right]$, where $l_{0 i}$ is the total number of lines terminating at point $i$ (where $l_{0 i} \geq 0$ ), and $l_{1 i}$ is the total number of lines continuing at point $i$ (where $l_{1 i} \geq 0$ ). For the network in Figure 5, this gives $S^{=}=(L, K) ; L=\{\mathrm{A}, \mathrm{B}, \mathrm{C}, \mathrm{D}, \mathrm{E}\}$; $K=\left\{\mathrm{K}_{\mathrm{A}}, \mathrm{K}_{\mathrm{B}}, \mathrm{K}_{\mathrm{C}}, \mathrm{K}_{\mathrm{D}}, \mathrm{K}_{\mathrm{E}}\right\} ; \mathrm{K}_{\mathrm{A}}=\{[2,0],[0,2],[1,0]\} ; \mathrm{K}_{\mathrm{B}}=\{[2,0],[2,1],[1,0]\} ; \mathrm{K}_{\mathrm{C}}=\{[1,0],[0,2],[1,1],[1,0]\} ;$ $\mathrm{K}_{\mathrm{D}}=\{[2,1],[1,1]\} ; \mathrm{K}_{\mathrm{E}}=\{[2,1],[1,0]\}$ (fig. 9).

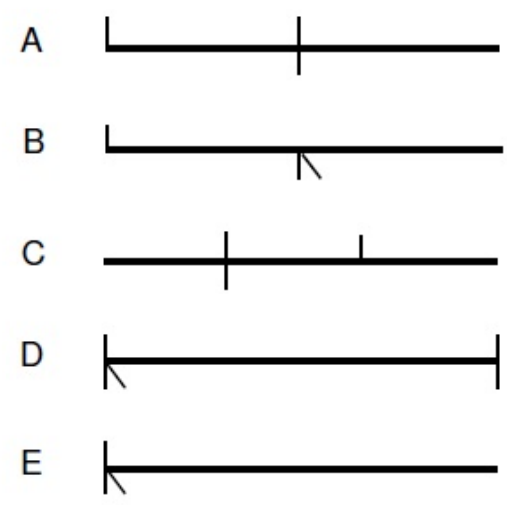

Figure 9: The line set $S^{=}$of continuity and termination conditions for the lines in the line structure of Figure 5

Figure 9 is not a graph but a series of line structure components, where each bold horizontal line represents the line in question, and the fine vertical or diagonal line stubs represent parts of other lines. In effect, this information equates with the specification of individual routes in route structure analysis (Marshall 2005) (therein defined graphically but not explicitly in terms of continuity and termination conditions).

Note that from $S^{=}$it is not possible to generate a uniquely corresponding line structure $S^{\#}$, without further information. For example, $S^{=}$does not tell us which other lines a given line connects with; for this we would need $G^{\prime}$, but $G^{\prime}$ ' does not tell us where those lines connect (e.g. at the beginning, middle, or end, etc). Hence we need to consider the overall relation with $S^{\#}$.

\subsection{Relations between $S^{\#}, G^{\prime}, G^{\prime \prime}$, and $S^{=}$}

We can set out fully the information contained in $S^{\#}, G, G^{\prime \prime}$ and $S^{=}$(Table 2). From the foregoing it can be seen that the line structure $S^{\#}$ amounts to the sum of information contained in $G$, $G^{\prime \prime}$ and $S^{=}$, i.e.,

$S^{\#}=G^{\prime} \cup G^{\prime} \cup S^{=}$.

This is an interesting and significant finding, as it demonstrates the tightly fit relationship between the three kinds of structure: (i) the primal $G^{\prime}$ and dual $G^{\prime}$ ' graphs are mutually exclusive or complementary; (ii) 
they express information found in $S^{\#}$; (iii) yet none of $G$, $G^{\prime \prime}$ nor $G^{\prime} \cup G^{\prime \prime}$ are enough to obtain $S^{\#}$; (iv) the "missing" element is "continuity and termination conditions" (CTC), this is supplied by $S^{=}$; (v) together these three make up the equivalent of $S^{\#}$. This indeed brings home what route structure analysis offers that is missing from primal and dual approaches, while showing how route structure analysis (via its implicit use of line structure) incorporates everything contained in $G$ and $G^{\prime}$.'

The practical significance of this is that the line structure embodies properties that are not present in either the primal graph or the dual graph. As we shall see, these properties are to do with continuity and termination and their relation to role in the road hierarchy. Let us now consider what these properties are.

\section{$5 \quad$ Properties of line structures}

In this section we first demonstrate hierarchical properties that the line structure captures directly that the primal graph and the dual graph do not. These are (i) continuity; (ii) junction type; and (iii) cardinality, a new indicator of hierarchical status. These are properties that can differentiate the relative importance of different lines in a line structure, and the hierarchical nature of a line structure as a whole, which may by extension be applied to route structures and road networks. Finally, we consider the issue of matrix size requirements for specifying line structures, relative to equivalent graphs.

\subsection{Continuity}

Some lines continue through intersections while others terminate, so we can distinguish lines by their continuity. For example, in the tree structure of Figure 8(d), the trunk (line A) is continuous through three intersections, while line $\mathrm{C}$ does not continue through any intersection. Primal graph representations necessarily cannot directly capture this property of continuity, because the linear elements are analyzed as discrete line segments which do not continue through the vertices representing intersections (Figure 8g, h, i). While some dual approaches may in fact "aggregate" or "concatenate" individual links or axial lines or line segments into more continuous entities (e.g., Thomson and Richardson 1999; Turner 2007; Jiang 2007; Tomko, Winter, and Claramunt 2008), the continuity itself is typically not explicitly calculated; continuity is in any case not obtainable from the dual graph, of itself (Figure 8j, k, l).

However, the line structure (or line set) can distinguish the continuity of lines. Indeed, in route structure analysis, continuity $(l)$ is simply identified as the number of line segments that a route is constituted by; this makes it a simple and convenient indicator that can be identified by visual inspection from a network diagram (Marshall 2005). In Figure 8 (d), line A comprises four line segments and so (in route structure analysis terms) has a continuity $(l)$ of 4 . Line B has a continuity of 2 , while lines $C, D$ and $E$ each has a continuity of 1.

\section{$5.2 \quad$ Junction type}

In graph-based analyses of road networks, junction type is typically considered in terms of nodal degree: In a primal graph, a node of degree 3 can be equated with a $T$-junction and a node of degree 4 can be equated with an X-junction (crossroads). For example in the network in Table 2, node 7 has degree 3, while nodes 2 and 4 have degree 4 . However, there is more to junction type than nodal degree. Some roads might be continuous through a junction, while others terminate. We can also recognize these "continuity and termination conditions" as being part and parcel of network structure, though these are not routinely captured in most road network analyses (Table 3).

The primal graph $G$ does not represent continuity of lines through intersections, and therefore could not distinguish between a $\mathrm{T}$ junction and a $\mathrm{Y}$ junction, or between an $\mathrm{X}$ junction and a $\mathrm{K}$ junction. Meanwhile, 
the dual graph representation $G^{\prime \prime}$ cannot capture the continuity and termination conditions of lines that intersect. For example, it cannot differentiate between cases with two lines (L, $\mathrm{T}$ or $\mathrm{X}$ ) or between three lines $(\mathrm{Y}$ or $\mathrm{K}$ or six-pointed star). However, a line structure (or line set or route structure) can capture the distinction between intersection type on the basis of differential continuity and termination: The line structure $S^{\#}$ can be seen to differentiate L, T, Y, X, K, and * junction types (Table 3).

Table 3: Junction types represented as line structures $\left(S^{*}\right)$, primal graphs $\left(G^{\prime}\right)$ and dual graphs $\left(G^{\prime}\right)$. Only $S^{\#}$ can uniquely distinguish between the six types of junction.

\begin{tabular}{ll}
\hline Junction type & $S^{\#}$ \\
$\mathrm{~L}$ (2 roads joining) \\
$\mathrm{K}$ (3 roads meeting)
\end{tabular}

\subsection{Hierarchy and cardinality}

Although a sense of hierarchy could be obtained by ranking roads by their connectivity (the degree of vertices in the dual graph) or indeed by their continuity (Section 5.1), conventional network analyses give less attention to the kind of hierarchy relating to differential continuity and termination-where primary roads are continuous through junctions, while subsidiary roads terminate, and hence where there is a direct hierarchical relation established between the continuing and terminating roads.

Primal graph approaches necessarily cannot represent hierarchy of this kind, because links are not continuous through intersections (cf Figure 8g-i; Table 2 column ii). Dual graph approaches necessarily cannot represent hierarchy of this kind because while the dual graph features lines as continuous entities, the dual graph does not capture the asymmetrical nature of the relations between the elements: which lines continue and which terminate in relation to each other (cf Figure 8j-1; Table 2 column iii). A line structure, however, can distinguish this kind of hierarchy associated with differential continuity and termination. For example, in the tree structure in Figure 8d, the trunk (line A) is continuous through its intersections with branches B, C, and D. Branches B, C, and D terminate on A. Meanwhile, line E terminates on B. This can be interpreted as A having a higher hierarchical status than $\mathrm{B}, \mathrm{C}$, and $\mathrm{D}$, and $\mathrm{B}$ having a higher status than $\mathrm{E}$. This gives a sense of hierarchy, which could be expressed mathematically as partial order relations: $E \leq B ; B \leq A, C \leq A, D \leq A$. (Here we can conclude that $\mathrm{E} \leq \mathrm{A}$; the partial order equations allow us to make inferences across the network, between roads that are not directly connected.) 
Indeed, in general we could interpret hierarchy based on differential continuity and termination in the following terms: (1) where a line yields - that is, terminates on a line (or lines) prevailing though an intersection-then the yielding line is of lower (or equal) status compared with the prevailing line(s) (e.g., in Figure 5, D yields on $\mathrm{C} ; \mathrm{D} \leq \mathrm{C})$; (2) where two lines intersect without termination, neither is deemed to yield to the other, and no conclusions are drawn on their relative hierarchical status (e.g., lines A and C in Figure 5); (3) where two lines terminate at the same point, neither is deemed to yield to the other, and no conclusions are drawn on their relative hierarchical status (e.g., lines A and B in Figure 5). Note that we are distinguishing here between a line that terminates (comes to an end) and one that yields (terminates where at least one other line prevails); and between a line that continues (passes through a point without terminating) and one that prevails (continues where at least one other line terminates).

While this can give the relative hierarchical ordering between elements (as already seen above for Figure 8, $\mathrm{E} \leq \mathrm{B}$ etc.), it would be useful to be able to quantify more precisely the hierarchical value for each line, in a way that can distinguish more finely between the roles of different lines in a line structure. There are several possible ways of doing this. Here, we create a property that relates to the way that a prevailing line has a superior status to that of yielding lines. Hence we define a simple property called cardinality $(k)$ as follows: (1) each line has a cardinality value $(k)$ equal to one more than the highest $k$ value of lines that yield to it; (2) a line with no lines yielding to it has a cardinality of 1 . In the tree structure in Figure $8(\mathrm{~d})$, lines $\mathrm{C}, \mathrm{D}$, and $\mathrm{E}$ each has a cardinality of 1 ; line $\mathrm{E}(k=1)$ yields on $\mathrm{B}$, so $\mathrm{B}$ has a cardinality of 2 ; of all the lines yielding on $\mathrm{A}$, line $\mathrm{B}$ has the highest $k$ value (2), so A has a cardinality of $2+1=3$. Cardinality can also be applied to grid structures, to express hierarchical distinction between prevailing and yielding lines, where these distinctions are salient. In general, cardinality values can be used to compare the relative hierarchical status of lines in any line structure.

\subsection{Information considerations for matrix specification}

The amount of information required to specify a line structure depends, at least, on the number of lines $(L)$ present; plus, in the case of the ordinal point matrix, the number of points $(P)$ present; or in the case of the parametric point matrix, the number of intersection points present $(I)$ :

Parametric point matrix: $I \times L$

Parametric line matrix: $L \times L$

Ordinal point matrix: $P \times L$

Ordinal line matrix: $L \times L$

For the corresponding primal graph (Figure 10), the information required would typically be represented in the form of an incidence matrix $(V \times E)$ or adjacency matrix $(V \times V)^{9}$.

9 For brief descriptions of these, see, for example, http://mathworld.wolfram.com/IncidenceMatrix.html; http://mathworld.wolfram.com/AdjacencyMatrix.html. 


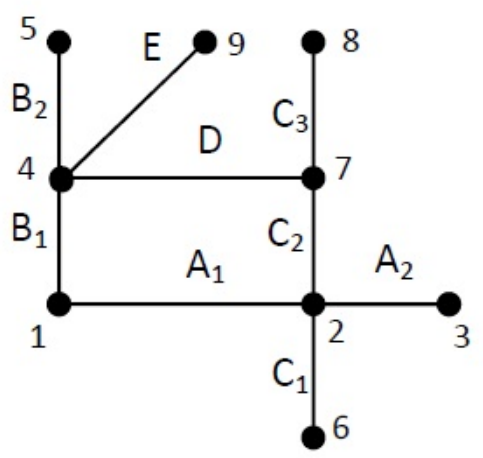

Figure 10: Primal graph corresponding to Figures 5, 6 and 7.

The incidence matrix $(V \times E)$ for the network of Figure 10 would be:

\begin{tabular}{|c|c|c|c|c|c|c|c|c|c|}
\hline & $A_{1}$ & $A_{2}$ & $B_{1}$ & $B_{2}$ & $C_{1}$ & $C_{2}$ & $C_{3}$ & $D$ & $E$ \\
\hline 1 & & 0 & 1 & 0 & 0 & 0 & 0 & 0 & 0 \\
\hline 2 & 1 & 1 & 0 & 0 & 1 & 1 & 0 & 0 & 0 \\
\hline 3 & 0 & 1 & 0 & 0 & 0 & 0 & 0 & 0 & 0 \\
\hline 4 & 0 & 0 & 1 & 1 & 0 & 0 & 0 & 1 & 1 \\
\hline 5 & 0 & 0 & 0 & 1 & 0 & 0 & 0 & 0 & 0 \\
\hline 6 & 0 & 0 & 0 & 0 & 1 & 0 & 0 & 0 & 0 \\
\hline 7 & 0 & 0 & 0 & 0 & 0 & 1 & 1 & 1 & 0 \\
\hline 8 & 0 & 0 & 0 & 0 & 0 & 0 & 1 & 0 & 0 \\
\hline 9 & 0 & 0 & 0 & 0 & 0 & 0 & 0 & 0 & 1 \\
\hline
\end{tabular}

The adjacency matrix $(V \times V)$ for the network of Figure 10 would be:

\begin{tabular}{|c|c|c|c|c|c|c|c|c|}
\hline 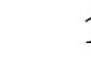 & 2 & 3 & 4 & 5 & 6 & 7 & 8 & 9 \\
\hline 1 & 1 & 0 & 1 & 0 & 0 & 0 & 0 & \\
\hline 2 & 0 & 1 & 0 & 0 & 1 & 1 & 0 & 0 \\
\hline 3 & 1 & 0 & 0 & 0 & 0 & 0 & 0 & 0 \\
\hline 4 & 0 & 0 & 0 & 1 & 0 & 1 & 0 & 1 \\
\hline 5 & 0 & 0 & 1 & 0 & 0 & 0 & 0 & 0 \\
\hline 6 & 1 & 0 & 0 & 0 & 0 & 0 & 0 & \\
\hline 7 & 1 & 0 & 1 & 0 & 0 & 0 & 1 & 0 \\
\hline 8 & 0 & 0 & 0 & 0 & 0 & 1 & 0 & 0 \\
\hline 9 & 0 & 0 & 1 & 0 & 0 & 0 & 0 & \\
\hline
\end{tabular}

Clearly, these line structures and graphs have different data requirements. For the network in Figures 5-7, the matrices for specifying the line structures (Section 3.3) are smaller than those used for specifying the corresponding graphs (Figure 10). Of course, the data requirements-and the degree of potential saving in matrix size-would depend on the nature of the network concerned.

Figure 11 shows a range of seven example networks for consideration. The simplest comparison of matrix size, only involving two contrasting variables, would be to compare the graph's adjacency matrix (involving only $V^{2}$ - squared since the matrix is two dimensional) and one of the line matrices (involving only $L^{2}$ ). The 
smaller the value of $L^{2}$ relative to $V^{2}$, the greater the extent to which the line structure (line matrix) specification will be the more parsimonious specification. ${ }^{10}$

The range of values for the networks in Figure 11 are given in Table 4. In most cases, the amount of information to specify the line structure is less than that to specify the graph-often considerably less. In particular, those cases that may be considered most like road networks are (c), (d), (e) and (f)—featuring a mix of circuits, pendant lines or edges $(V>I)$, and lines continuous through intersections $(L<E)$ - show $L^{2} / V^{2}$ values in the range 0.25 to 0.64 . Those considered less like road networks ( $b$ and g) because of their discontinuity are the only cases with any of these ratios equaling or exceeding 1 . The lowest values are found for case (a), which has no three-way intersections-as such this case is less typical of road networks in general, though it could represent some grids.

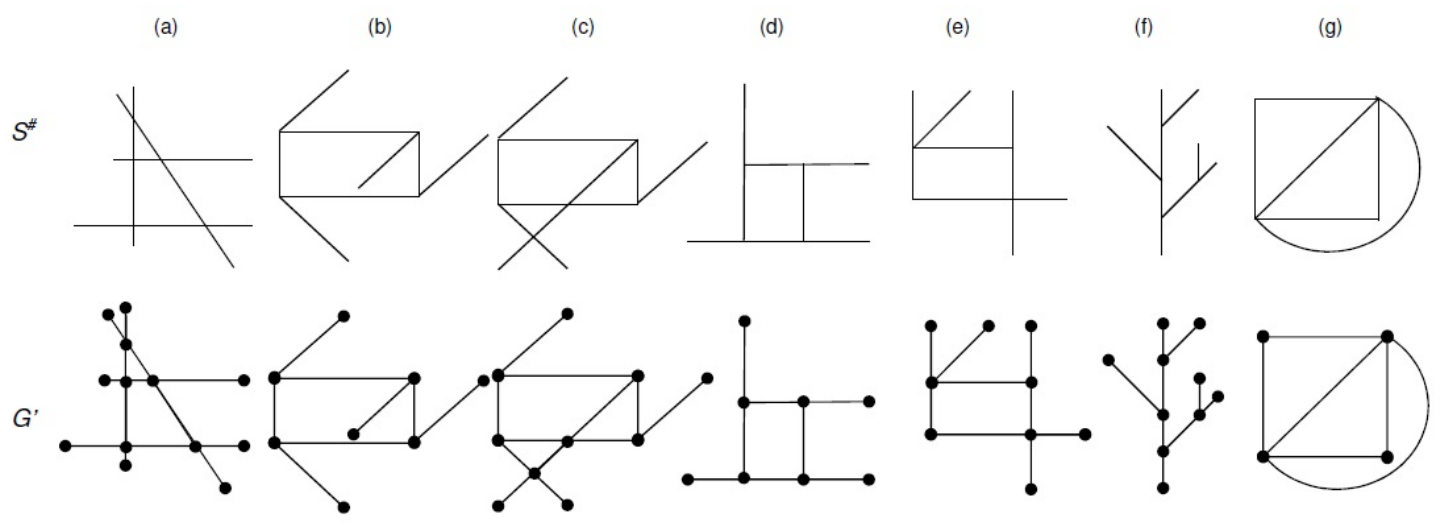

Figure 11: Example line structures $\left(S^{\#}\right)$ and corresponding network graphs $(G)$.

Table 4: Properties relating to matrix size requirements for the networks in Figure 11.

\begin{tabular}{lllllllll}
\hline Network & & $(\mathrm{a})$ & $(\mathrm{b})$ & $(\mathrm{c})$ & $(\mathrm{d})$ & $(\mathrm{e})$ & $(\mathrm{f})$ & $(\mathrm{g})$ \\
\hline Intersections & $\mathrm{I}$ & 5 & 4 & 6 & 4 & 4 & 4 & 4 \\
Lines & $\mathrm{L}$ & 4 & 8 & 8 & 4 & 5 & 5 & 6 \\
Vertices & $\mathrm{V}$ & 13 & 8 & 10 & 8 & 9 & 10 & 4 \\
Edges & $\mathrm{E}$ & 14 & 8 & 12 & 8 & 9 & 9 & 6 \\
Ratio & $\mathrm{L}^{2} / \mathrm{V}^{2}$ & 0.09 & 1.0 & 0.64 & 0.25 & 0.31 & 0.25 & 2.25 \\
\hline
\end{tabular}

We can also make some theoretical calculations for larger networks (Figure 12). This, (together with Figure 11 and Table 4) confirms that networks with more pendant nodes, and more continuous lines, and fewer multi-spoke vertices, are more likely to be more concisely specified by line structures. This effect is likely to be increasingly pronounced with network size for grid-like networks. For the tree comprising eight lines (Figure 12c), the value of $L^{2} / V^{2}$ is 0.25 ; for an equivalent network comprising 16 lines, $V$ would be 32, and $L^{2} / V^{2}$ would remain at 0.25 . However, for the grid of Figure $12 \mathrm{~d}$, increasing $L$ to 16 would increase $V$ to 64 ; and $L^{2} / V^{2}$ would reduce to 0.06 ; for the grid of Figure $12 \mathrm{f}$, increasing $L$ to 16 would increase $V$ to 96 , and $L^{2} / V^{2}$ would reduce to 0.03 .

10 Alternatively, we could use IL/EV, involving all four variables, where the smaller the value of IL/EV, the greater the extent to which the line structure (line matrix) will have the more parsimonious specification. In this case, by their mathematical definition, $I \leq V$ and $L \leq E$. Hence IL/EV $\leq$. In other words, if comparing incidence matrices of a graph versus the parametric point matrix, the latter will always be equal to or smaller than the former. 
(a)
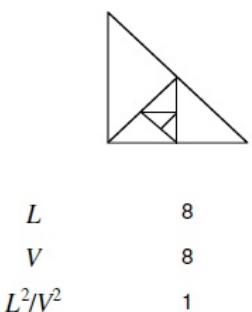

(b)

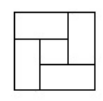

\section{8}

12

0.44 (c)

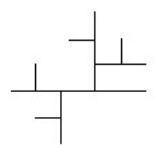

8

16

0.25 (d)

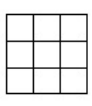

8
16
0.25

(e)
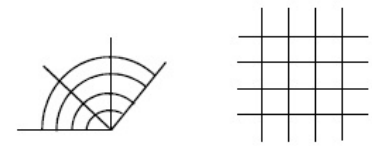

8

32

0.06

Figure 12: $L^{2} / V^{2}$ values for larger theoretical networks.

The conclusion here is that in terms of matrix size, the line structure specification is likely to be more parsimonious than graph specification for road networks with some roads continuous through junctions, and increasingly so with network size for grid-like networks. That said, the practical utility of this in data management and computational terms would depend on other factors to do with detailed specification within proprietary software platforms, which may in any case incorporate graph-theoretical measures such as $V$ and/or $E$, or which may not store network specification in this kind of matrix format. Nevertheless, this section has shown that line structures can, in principle, represent more network properties with less data. This invites further scrutiny beyond this paper. For now we turn to demonstration of application to road networks.

\section{Application to road network structure}

The properties set out in the previous section could be applied to any real-world system representable as a line structure. We now demonstrate the application of these properties to the road system context. Here, we look at two small networks, based on real street layouts for illustrative purposes: one represents a small village network (Figure 13, left), the other a portion of an inner city grid (Figure 13, right). These are represented as line structures (a, b), primal graphs (c, d), and dual graphs (e, f). As a check, the values of $L^{2} / V^{2}$ for these networks can be calculated: these are 0.30 for the village network and 0.27 for the city grid. These fall comfortably within the range of the values for the "most road network like" networks in Figure 11c, d, e, and f. 


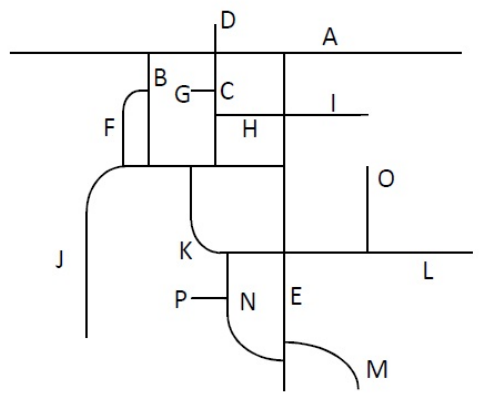

(a) Line structure $(L=16)$

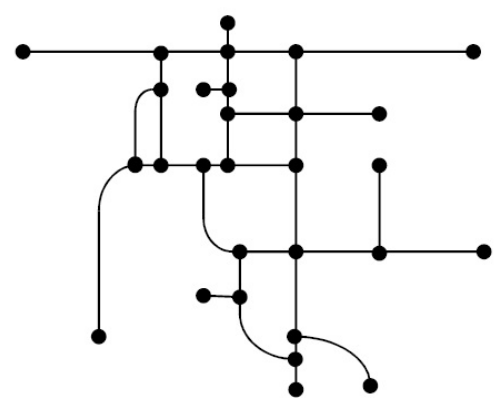

(c) Primal graph $(V=29)$

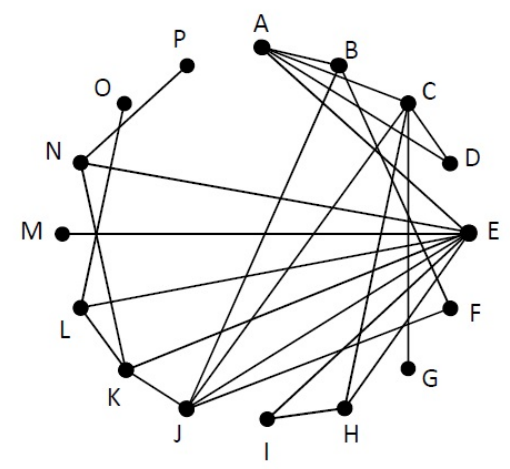

(e) Dual graph

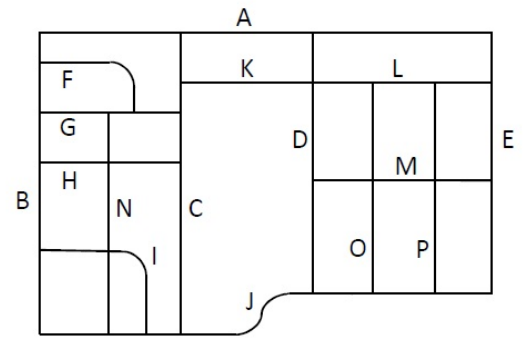

(b) Line structure $(L=16)$

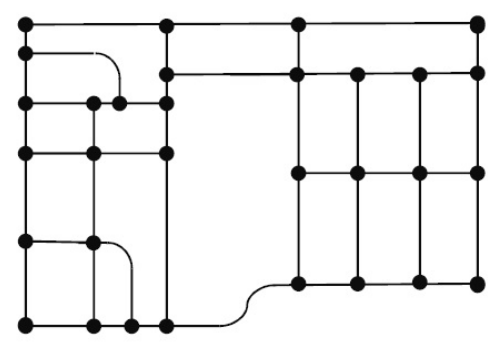

(d) Primal graph $(V=31)$

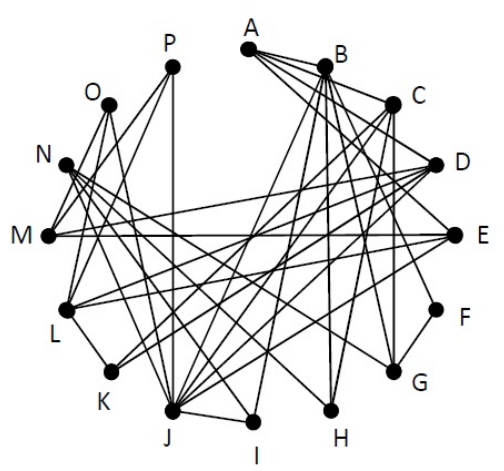

(f) Dual graph

Figure 13: Two example road networks. Left-hand side: Village network. Right-hand side: City grid network.

\subsection{Hierarchical differentiation of routes}

Each line in each network has values calculated in terms of continuity, connectivity, and cardinality (Table 5a and b). For example, for the village network (Figure 13a), line $\mathrm{K}$ has a continuity of 2 (it is composed of two individual line segments); it has a connectivity of 4 (as it connects with four other lines, namely E, J, L, and $\mathrm{N})$. The cardinality value is calculated as 3 , since it prevails over line $\mathrm{N}$, which has a cardinality of 2 , which value is derived in turn since line $\mathrm{N}$ prevails over line $\mathrm{P}$, which has a cardinality of 1 (as it prevails over no other line). 
Lines can then be ranked according to any of these properties, i.e. continuity or connectivity, or cardinality. For example, in the case of the village network (Figure 13a, Table 5a), the three most important lines emerging are A, E and J. For both continuity $(l)$ and connectivity $(c)$, E has the highest value, above that of J and A. But for cardinality $(k), \mathrm{A}$ is the highest, followed by $\mathrm{E}$ and $\mathrm{J}$. The cardinality value picks up that $\mathrm{E}$ is the locally most connective road of the village, while A is a strategic through route, which $\mathrm{E}$ terminates on. Hence cardinality can generate an alternative basis for hierarchical distinction other than simply the differential connectivity or continuity.

In the case of the city grid (Figure 13b, Table 5b), we can see how $\mathrm{J}$ is clearly the most continuous and most connective road, followed by B and C (and others). However, the relative significance of A is indicated by its cardinality value, $k$. A has the highest equal $k$ value (4), which places it above both $\mathrm{B}$ and $\mathrm{C}$. What $k$ is picking up is that while $\mathrm{A}$ only has two junctions along it, those junctions are with locally significant roads (C and D), which themselves gather up the most local roads. Route A therefore has the character of a strategic road with fewer but more significant intersections along it. The cardinality indicator has the advantage that the value relates to the rest of the network; a line gains in status not just because of the presence of side roads immediately off it, but also because of all lines within its "yield catchment."

Table 5 (a): Properties of line structures in village network (fig. 13 a)

\begin{tabular}{llll}
\hline Line & Continuity & $\begin{array}{l}\text { Connectivity } \\
(c)\end{array}$ & $\begin{array}{l}\text { Cardinality } \\
(k)\end{array}$ \\
\hline A & $(l)$ & 4 & 6 \\
B & 4 & 3 & 2 \\
C & 2 & 5 & 2 \\
D & 3 & 2 & 1 \\
E & 1 & 8 & 5 \\
F & 6 & 2 & 1 \\
G & 1 & 1 & 1 \\
H & 1 & 3 & 1 \\
I & 1 & 2 & 1 \\
J & 1 & 5 & 4 \\
K & 5 & 4 & 3 \\
L & 2 & 3 & 2 \\
M & 2 & 1 & 1 \\
N & 1 & 3 & 2 \\
O & 2 & 1 & 1 \\
P & 1 & 1 & 1 \\
\hline
\end{tabular}


Table 5 (b): Properties of line structures in city grid network (fig. 13 b)

\begin{tabular}{llll}
\hline Line & $\begin{array}{l}\text { Continuity } \\
(D\end{array}$ & $\begin{array}{l}\text { Connectivity } \\
(c)\end{array}$ & $\begin{array}{l}\text { Cardinality } \\
(k)\end{array}$ \\
\hline A & 3 & 4 & 4 \\
B & 5 & 6 & 3 \\
C & 4 & 5 & 3 \\
D & 3 & 5 & 3 \\
E & 3 & 4 & 3 \\
F & 1 & 2 & 1 \\
G & 3 & 4 & 2 \\
H & 2 & 3 & 1 \\
I & 2 & 3 & 1 \\
J & 7 & 8 & 4 \\
K & 1 & 3 & 1 \\
L & 3 & 5 & 2 \\
M & 3 & 4 & 1 \\
N & 3 & 4 & 1 \\
O & 2 & 3 & 1 \\
P & 3 & 3 & 1 \\
\hline
\end{tabular}

\subsection{Hierarchical differentiation of junction type}

The line structure representation can differentiate junction type more sensitively than either the primal graph or dual graph (Table 6). For example, in the city grid network (Figure 12b), the lines $\mathrm{K}$ and $\mathrm{L}$ are identified as two separate lines that terminate on line $\mathrm{D}$; as such this is considered a " $\mathrm{K}$ " junction, whereas line $\mathrm{M}$ is deemed to "cross over" lines $\mathrm{O}$ and $\mathrm{P}$ without any of these lines terminating (i.e., interpreted as X junctions). Overall, Table 7 demonstrates how the line structure representation provides precise information about the types of junctions present: $\mathrm{L}, \mathrm{T}, \mathrm{Y}, \mathrm{X}, \mathrm{K}$, or * which cannot be uniquely specified by $G$ or $G^{\prime}$ ' alone. 
Table 6: Junction types identified by different network representations

\begin{tabular}{llll}
\hline & Junction type & Village network & City grid network \\
\hline Line structure $S^{\#}$ & & Fig. 13 (a) & Fig. 13 (b) \\
& $\mathrm{L}$ & 0 & 4 \\
& $\mathrm{~T}$ & 15 & 22 \\
$\mathrm{X}$ & 3 & 4 \\
& $\mathrm{Y}$ & 0 & 0 \\
$\mathrm{~K}$ & 0 & 1 \\
& $*$ & 0 & 0 \\
Primal graph $G$, & Fig. 13 (c) & Fig. 13 (d) \\
& L (degree 2) & 0 & 4 \\
& T or Y (degree 3) & 15 & 22 \\
X or K (degree 4) & 3 & 5 \\
Dual graph $G$, & $*$ (degree 6) & 0 & 0 \\
& & Fig. 13 (e) & Fig. 13 (f) \\
& L or T or X (degree 2) & 18 & 30 \\
& Y or K or * (degree 3) & 0 & 1 \\
\hline
\end{tabular}

\subsection{Hierarchical differentiation of networks}

We can also use the cardinality values to compare and contrast the structure of whole networks to interpret whether they are more or less hierarchical. Here we can create a "cardinality graph," $G_{\mathrm{K}}$ ", showing the yield relations between each line in a line structure (Figure 14). The cardinality graph $G_{\mathrm{K}}$ " (Figures 14a, b) is a subset of the equivalent dual graph $G^{\prime}$ ' (Figures $13 \mathrm{e}, \mathrm{f}$ ), where only yield-relationships are shown (i.e., as occur at $\mathrm{T}$ or $\mathrm{K}$ junctions: where one line yields on another).

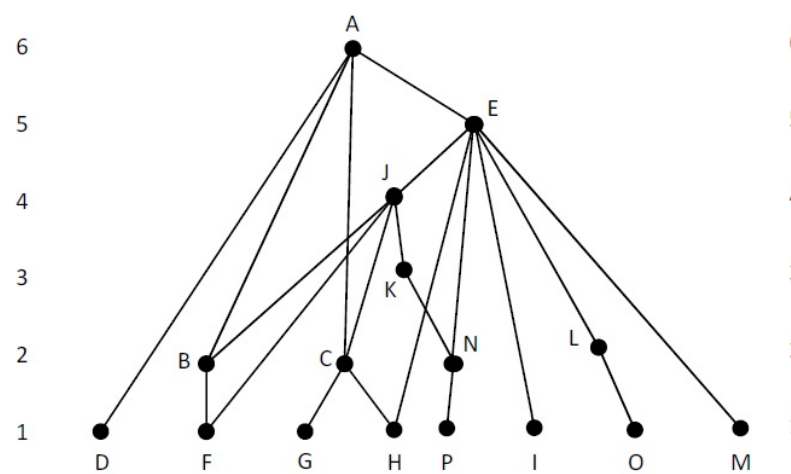

(a)

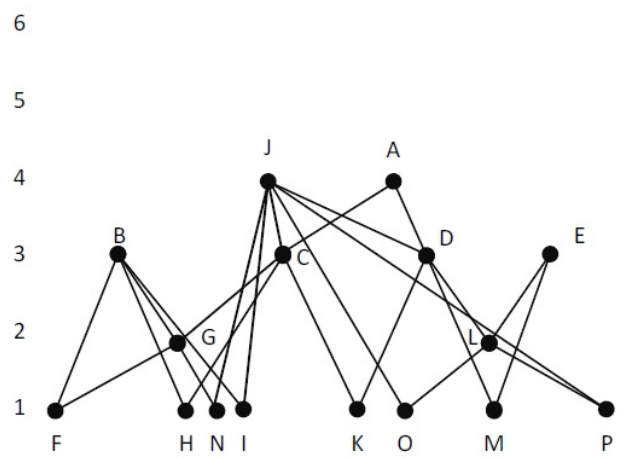

(b)

Figure 14: Cardinality graph $\left(G_{\mathrm{K}}{ }^{\prime \prime}\right)$ for (a) village network; (b) city grid; showing yield relations, ordered vertically by cardinality ( $k$ value). Each $G_{\mathrm{K}}$ " is a subset of the respective dual graphs shown in Figure 13 (e) and (f). 
We can immediately see that the cardinality graphs $G_{\mathrm{K}}$ " graphically differentiate the two networks. The graph for the village network (Figure 14a) comes to a single peak, whereas that for the city grid network (Figure 14b) is dissipated into four separate peaks. For the village network, the maximum cardinality value is absolutely higher (6 versus 4), and by calculation, the weighted average is also higher $(2.125$ versus 2.0 respectively). Therefore cardinality provides a simple quantitative indication of how one network can be "more hierarchical" than the other (i.e., over and above being "more connected").

The significance here is that these indicators of hierarchy are obtainable through recognizing yield relations, and none of which would be obtainable simply from the conventional primal graph or dual graph. (While $G_{\mathrm{K}}$ " is a subset of $G^{\prime \prime}$, the former cannot be obtained directly from the latter, but needs additional information from $S^{=}$or $S^{\#}$ ). So, however we may wish to define hierarchical structure, it seems useful to have these indicators available, and not simply overlook them due to use of conventional graph representation.

\subsection{Potential application to network design and management}

In addition to network science (analysis and modeling), line structures and their related hierarchical properties might be applied as part of network design and management, such as in the creation or specification of hierarchies, or analysis of prospective hierarchical structures. Any of the properties of continuity, connectivity, or cardinality, suitably ranked or combined, could be used to create a formal hierarchy of roads. We have already seen that cardinality can create a ranked series of roads in a network. For example, given the original line structures (Figure 13a, b), one could create a hierarchy where each tier corresponded to a cardinality value. Hence the village network would have six tiers (Figure 14a), while the city grid network would have four tiers (Figure 14b).

The pattern of roads with their cardinality values gives a visual impression of the hierarchical structure of a network (Figure 15). The interesting point to note here is that the relation to "arteriality", by which the set of main roads down to any given level all connect up contiguously (Morrison 1966, Marshall 2005). Arteriality will apply here, at least locally, in relation to the set of non-yielding roads. If each non-yielding road (for village, line A; for city grid, lines A, B, E, and J) is taken as the top tier in its own hierarchy, and all others ranked by cardinality, then the set of all roads down to any given level will be a single contiguous network. This is guaranteed because where a road $\mathrm{X}$ yields on a road $\mathrm{Y}$, road $\mathrm{Y}$ will always have a cardinality value greater than or equal to that of X. Hence cardinality could be used to generate "automatically" a hierarchy of main roads and subsidiary roads that makes sense spatially in terms of arteriality. This could be applied to any network (urban or rural, tree-like or grid-like) of any size.

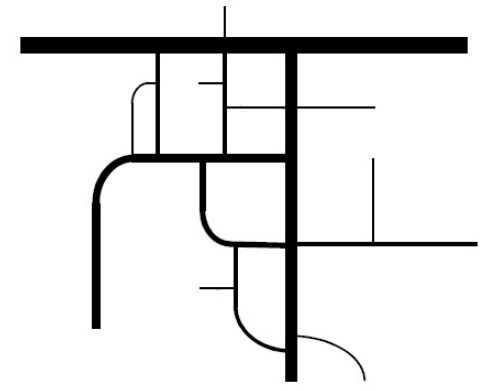

(a)

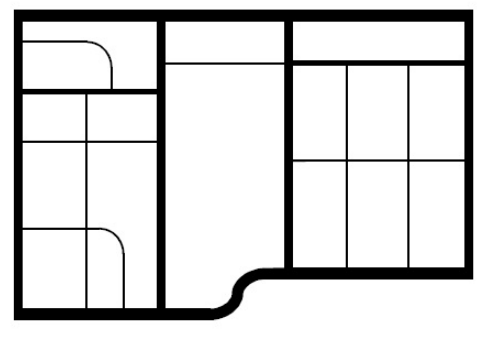

(b)

Figure 15: Line structures weighted by cardinality values can be related to arteriality: (a) village; (b) city grid. 


\section{$7 \quad$ Conclusions}

This paper has reached five primary results of significance for the articulation of road network structure. First, the paper has demonstrated the line structure itself_-identifiable as a linearly ordered incidence structure-as a mathematical entity $\left(S^{*}\right)$ that can take both discrete and continuous forms $\left(S_{\mathrm{O}^{*}}\right)$ and $\left(S_{\mathrm{P}}{ }^{*}\right)$ and can be represented by incidence matrices. The line structure can be seen to underpin route structure and other linear representations of road networks, bridging between cartographical, geometric, and graph theoretical representations of road networks (Table 7). 
Table 7: A spectrum of road layout representations

Graphic representation Explanation Properties

(a) Map

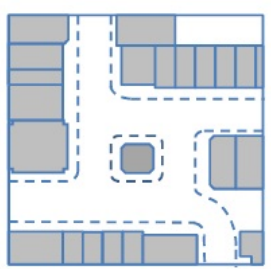

(b) Abstraction of 2-

D geometry

(polygon)

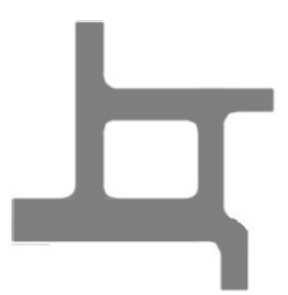

Continuous two-dimensional road surface (polygon)

Continuous two-dimensional

Areas

surface (polygons)

Length

Breadth

Angles

Road areas

Road length

Road breadth

Angles

Centrelines with absolute

Road lengths

geographical location, metric

Angles

length and absolute orientation;

and continuity

Identity of lines continuous

through intersections

All points along lines (including points between intersections)

Identity of lines continuous

through intersections

End points and intersection

points only

Discrete links or edges

(discontinuous)

Discrete nodes or vertices (end points and intersection points only)
Connectivity, continuity and cardinality

Any properties associated with graphs (see below)

Degree of nodes or vertices; connectivity; network distance;

coefficient of clustering, Beta index, etc. 
Second, the paper has explicitly identified the line set $S^{=}$whose continuity and termination conditions (CTC) can be seen as being necessary and significant in filling a mathematical gap between $S^{\#}$ and $G$ and $G^{\prime \prime}$ : hence the fundamental relation $S^{\#}=G^{\prime} \cup G^{\prime} \cup S^{=}$. Indeed, the CTC can be seen retrospectively as the "raw material" from which hierarchical properties such as continuity and cardinality are formed.

Third, in doing the above, the paper has clarified relations between existing network representations. While $G$ and $G^{\prime \prime}$ are mutually exclusive mathematical structures, this does not mean that the associated primal and dual approaches to network analysis need be considered rival, mutually exclusive methods, but can be seen as alternative selective abstractions from $S^{\#}$ to emphasize one (sub)set of attributes rather than another. Meanwhile, route structure analysis can be seen as being integral to the union of primal, dual and line structure approaches.

Fourth, the paper suggests that the line structure $\left(S^{\#}\right)$ can in principle represent more properties, using less data than graphs. This potential parsimony could be of interest for application to existing network analyses, even if the additional network properties addressed in this paper were not desired. In practice, of course, any utilitarian advantage would depend on what data was already available and what kind of data format it was held in.

Finally, the paper has introduced a specific new indicator of hierarchical differentiation, cardinality $(k)$, and shown a way of linking from this—via the "cardinality graph" $\left(G_{K}{ }^{\prime \prime}\right)$ - to creating a ranking of routes in a network, and linked to a pattern of "arteriality." Hence the paper helps provide a link between "configurational structure" and "constitutional structure" (Marshall 2005).

This paper in effect provides a mathematical retrofit and clarification of relations between graph-based and route structure approaches. Indeed, route structure analysis can be extended to include the new property of cardinality. Together this can pave the way toward more consistent and comprehensive network analysis, with potential application also to network design and management.

Further work suggested is fuller mathematical specification of line structures in terms of sensitivities and generalization; further mathematical elaboration of the relationships between different kinds of structure; and consideration of the data format and availability to enable practical application of line structure representations to road network analysis. Further development could address the possible automatic generation of representation of line structures from geographic or other data. Additionally, there are questions on how to decide what a continuous road is, in the first place-such as in physical or administrative terms-for representation as a continuous line in a line structure.

Future research could involve empirical testing or modeling for the relative significance and sensitivity of continuity and cardinality values in relation to network operation and performance variables (e.g., traffic flow, transport modes, land use frontages, path choice algorithms, etc.) and for potential application to road network management.

Finally, the core part of this paper concerning line structures and their properties-Sections 3 and 4 in particular-potentially has a more general significance outside of the context of road networks. Line structures could in principle be used to represent other kinds of structure where continuity of linear elements through intersections is significant, such as engineering structures, where lines could represent beams and columns. 


\section{References}

AASHTO. 2001. A Policy on Geometric Design of Highways and Streets. Washington, DC: American Association of State Highway and Transport Officials.

Banks, J. H. 1998. Introduction to Transportation Engineering. Boston: McGraw-Hill.

Barnes, J. A., and F. Harary. 1983. Graph theory in network analysis. Social Networks 5(2): 235-244.

Barthélemy, M., and A. Flammini. 2008. Modeling urban street patterns. Physical Review Letters 100: 138702.

Barthélemy, M. 2011. Spatial networks. Physics Reports 499: 1-101.

Batty, M. 2004. A new theory of space syntax. Working Paper 75. London: Center for Advanced Spatial Analysis, UCL.

Batty, M., and S. Rana. 2004. The automatic definition and generation of axial lines and axial maps. Environment and Planning B: Planning and Design 31(4): 615-640.

Batty, M. 2008. Whither network science? Environment and Planning B: Planning and Design 35: 569-571.

Batty, M. 2010. As simple as possible: Styles of model, styles of science. Environment and Planning B: Planning and Design 37: 1-2.

Batty, M. 2014. The New Science of Cities. Cambridge, MA: The MIT Press.

Bejan, A. 1996. Street network theory of organization in nature. Journal of Advanced Transportation 30(2): 85-107.

Bell, M. G. B., and Y. Iida. 1997. Transportation Network Analysis. Chichester, West Sussex: John Wiley.

Bredon, G. E. 1997. Topology and Geometry. New York: Springer-Verlag.

Brindle, R. 1996. Road hierarchy and functional classification. In Traffic Engineering and Management, edited by K. W. Ogden, and S. Taylor. Melbourne: Institute of Transport Studies, Department of Civil Engineering, Monash University.

Buckwalter, D. 2001. Complex topology in the highway network of Hungary, 1990 and 1998. Journal of Transport Geography 9: 125-135.

Buekenhout, F. (Ed.), 1995. Handbook of Incidence Geometry. Amsterdam: Elsevier Science.

Buekenhout, F., and A. M. Cohen. 2013. Diagram Geometry. Related to Groups and Buildings. Berlin and Heidelberg: Springer.

Buhl, J., J. Gautrais, N. Reeves, R. Solé, S. Valverde, P. Kuntz, and G. Theraulaz. 2006. Topological patterns in street networks of self-organized urban settlements. The European Physical Journal B 49: 513-522.

Cardillo, A., S. Scellato, V. Latora, and S. Porta. 2006. Structural properties of planar graphs of urban street patterns. Physical Review E 73: 066107.

Claramunt, C., and S. Winter. 2007. Structural salience of elements of the city. Environment and Planning B: Planning and Design 34: 1030-1050.

Clark, J., and D. A. Holton. 1991. A First Look at Graph Theory. Singapore: World Scientific.

Courtat, T., C. Gloaguen, and S. Douady. 2011. Mathematics and morphogenetics of cities: A geometrical approach. Physical Review E 83: 036106.

Deo, N. 1974. Graph Theory, with Applications to Engineering and Computer Science. New Delhi: Asohe K. Ghosh, Prentice Hall of India.

DfT and DCLG. 2007. Manual for Streets. London: Department for Transport and Department for Communities and Local Government.

Diestel, R. 2000. Graph Theory (2nd edition). New York: Springer-Verlag.

Erat, A., M. Löchl, and K. W. Axhausen. 2008. Graph-theoretical analysis of the Swiss road and railway networks over time. Networks and Spatial Economics 10.1007/s11067-008-9074-7

Essex County Council. 1973. A Design Guide for Residential Areas. Chelmsford, MA: Essex County Council. Faber, R. L. 1983. Foundations of Euclidean and Non-Euclidean Geometry. New York and Basel: Marcel Dekker.

Foulds, L. R. 1992. Graph Theory Applications. New York: Springer-Verlag. 
Fitzpatrick, R. 2008. Euclid's Elements in Greek. URL: http://farside.ph.utexas.edu/euclid/elements.pdf.

Gil, J. 2014. Analyzing the configuration of multimodal urban networks. Geographical Analysis 46: 368-391.

Godehardt, E. 1990. Graphs as Structural Models. The Application of Graphs and Multigraphs in Cluster Analysis (2nd edition). Braunschweig, Germany: Vieweg.

Gross, J., and J. Yellen. 1999. Graph Theory and its Applications. Boca Raton, FL: CRC Press.

Haggett, P., and R. J. Chorley. 1969. Network Analysis in Geography. London: Edward Arnold.

Hayes, B. 2000. Graph theory in practice: Part I. American Scientist 88(1): 9-13.

Hillier, B., and A. Penn. 2004. Rejoinder to Carlo Ratti. Environment and Planning B: Planning and Design 31: 501-511.

ICE. 1996. Which Way Roads? London: Thomas Telford Publishing on behalf of the Institution of Civil Engineers.

Jiang, B. 2007. A topological pattern of urban street networks: Universality and peculiarity. Physica A 384: 647-655.

Jiang, B. 2009. Street hierarchies: A minority of streets account for a majority of traffic flow. International Journal of Geographical Information Science 23(8): 1033-1048.

Jiang, B., and C. Claramunt. 2002. Integration of space syntax into GIS: New perspectives for urban morphology. Transactions in GIS 6(3): 295-309.

Jiang, B., and C. Claramunt. 2004a. A structural approach to the model generalization of an urban street network. Geoinformatica 8 (2): 157-171.

Jiang, B., and C. Claramunt. 2004b. Topological analysis of urban street networks. Environment and Planning B: Planning and Design 31: 151-162.

Jiang, B., and A. Okabe. 2014. Different ways of thinking about street networks and spatial analysis. Geographical Analysis 46: 341-344.

Jones, I. D. 1986. A review of highway classification systems. Traffic Engineering and Control 27(1): 27-30.

Jones, P., N. Boujenko, and S. Marshall. 2007. Link and Place. A Guide to Street Planning and Design. London: Landor Books.

Kansky, K. J. 1963. Structure of Transportation Networks: Relationships Between Network Geometry and Regional Characteristics. Chicago: University of Chicago, Department of Geography.

Karimi, K. 2012. A configurational approach to analytical urban design: 'Space syntax' methodology. Urban Design International 17(4): 297-318.

Lämmera, S., B. Gehlsena, and D. Helbinga. 2006. Scaling laws in the spatial structure of urban road networks. Physica A: Statistical Mechanics and its Applications 363(1): 89-95.

Lord, E. A., and C. B. Wilson. 1984. The Mathematical Description of Shape and Form. Chichester, West Sussex, UK: Ellis Horwood.

Marshall, S. 2005. Streets and Patterns. London and New York: Spon Press.

Masucci, A. P., D. Smith, A. Crooks, and M. Batty. 2009. Random planar graphs and the London street network. The European Physical Journal B 71(2): 259-271.

Masucci, P., Stanilov, K., and Batty, M. (2013) Exploring the Evolution of London's Street Network in the Information Space: A Dual Approach, arXiv:1311.7284v1 [physics.soc-ph] 28 Nov 2013.

Morlok, E. 1967. An Analysis of Transport Technology and Network Structure. Evanston: The Transportation Center, Northwestern University.

Morrison, A. 1966. Principles of road classification for road maps. Cartographic Journal 3(1): 17-30.

MoT. 1963. Traffic in Towns. London: HMSO.

Penn, A., B. Hillier, D. Banister, and J. Xu. 1998. Configurational modeling of urban movement networks. Environment and Planning B: Planning and Design 25: 59-84.

Peponis, J., J. Wineman, S. Bafna, M. Rashid, and S. H. Kim. 1998. On the generation of linear representations of spatial configuration. Environment and Planning B: Planning and Design 25: 559-576.

Porta, S., P. Crucitti, and V. Latora. 2006a. The network analysis of urban streets: A primal approach. Environment and Planning B: Planning and Design 33(5): 705-725. 
Porta, S., P. Crucitti, V. Latora. 2006b. The network analysis of urban streets: A dual approach. Physica A 369: 853-866.

Rosen, K. H. (Ed.). 2000. Handbook of Discrete and Combinatorial Mathematics. Boca Raton, FL: CRC Press.

Scellato, S., A. Cardillo, V. Latora, and S. Porta. 2006. The backbone of a city. The European Physical Journal B 50:221-225.

Strano, E., V. Nicosia, V. Latora, S. Porta, and M. Barthélemy. 2012. Elementary processes governing the evolution of road networks. Scientific Reports 2: 296.

Thomson, R. C., and D. E. Richardson. 1999. The 'good continuation' principle of perceptual organization applied to the generalization of road networks. Proceedings of ICA conference, Ottawa, August 14-21.

Tomko, M., S. Winter, and C. Claramunt. 2008. Experiential hierarchies of streets. Computers, Environment and Urban Systems 32(1): 41-52.

Turner, A., A. Penn, and B. Hillier. 2005. An algorithmic definition of the axial map. Environment and Planning B: Planning and Design 32(3): 425-444.

Turner, A. 2007. From axial to road-centre lines: A new representation for space syntax and a new model of route choice for transport network analysis. Environment and Planning B: Planning and Design 34(3): 539-555.

Wagner, R. 2008. On the metric, topological and functional structures of urban networks. Physica A: Statistical Mechanics and its Applications 387(8-9): 2120-2132.

West, D. B. 2001. Introduction to Graph Theory (2nd edition). Upper Saddle River, NJ: Prentice Hall.

Wilson, R. J. 2010. Introduction to Graph Theory (5th edition). Harlow: Prentice Hall.

Xie, F., and D. M. Levinson. 2007. Measuring the structure of road networks. Geographical Analysis 39: 336356.

Xie, F., and D. Levinson. 2011. Evolving Transportation Networks. New York: Springer.

Yerra, B. M., and D. M. Levinson. 2005. The emergence of hierarchy in transportation networks. The Annals of Regional Science 39(3): 541-553. 